\title{
The Quantum Geometry of Supersymmetry and the Generalized Group Extension Problem
}

\author{
Robert Oeckl* \\ Centre de Physique Théorique, CNRS Luminy, Case 907, \\ F-13288 Marseille - Cedex 9 \\ CPT-2001/P.4212 \\ 14 June 2001, 22 March 2002 (v2)
}

\begin{abstract}
We examine the notion of symmetry in quantum field theory from a fundamental representation theoretic point of view. This leads us to a generalization expressed in terms of quantum groups and braided categories. It also unifies the conventional concept of symmetry with that of exchange statistics and the spin-statistics relation. We show how this quantum group symmetry is reconstructed from the traditional (super) group symmetry, statistics and spin-statistics relation.

The old question of extending the Poincaré group to unify external and internal symmetries (solved by supersymmetry) is reexamined in the new framework. The reason why we should allow supergroups in this case becomes completely transparent. However, the true symmetries are not expressed by groups or supergroups here but by ordinary (not super) quantum groups. We show in this generalized framework that supersymmetry remains the most general unification of internal and space-time symmetries provided that all particles are either bosons or fermions.

Finally, we demonstrate with some examples how quantum geometry provides a natural setting for the construction of super-extensions, super-spaces, super-derivatives etc.
\end{abstract}

*email: oeckl@cpt.univ-mrs.fr 


\section{Introduction}

The question was raised a long time ago whether the external (space-time) and internal symmetries of the quantum field theories with which we describe nature could be part of a larger symmetry group that is not simply a direct product of the two.

For relativistic quantum mechanical theories the space-time symmetry group is the universal cover $\hat{P}$ of the Poincaré group $P$. (For simplicity we refer to $\hat{P}$ in the following as the Poincaré group.) Thus, a unification of symmetries in the abovementioned sense would imply a solution to the following problem: Is there a larger group $S \hat{P}$ which contains the Poincaré group $\hat{P}$, but is not simply a direct product of $\hat{P}$ and some other group? That is, is there a group $S \hat{P}$ with an inclusion

$$
\hat{P} \hookrightarrow S \hat{P} \quad \text { such that } S \hat{P} \neq \hat{P} \times G
$$

for any group $G$ ?

While mathematical solutions to the problem in this simple form can be easily found, they might not be of physical relevance. One can enlarge $\hat{P}$ for example by adding scale transformations. However, scale invariance is not a feature of the physically relevant quantum field theories of fundamental interactions. One therefore needs to impose additional constraints on $S \hat{P}$ in order for it to be physically interesting. Precisely such an analysis was carried out in the context of scattering theory in the 1960's, and brought into its most comprehensive form by Coleman and Mandula [1]. They were able to show that under reasonable physical assumptions the Lie algebra version of problem (11) has no solution: There is no such extension of the Poincaré Lie algebra.

Only a few years later, however, supersymmetry emerged as a physically acceptable solution to the extension problem in a modified from [2, 3]. One needs to extend the concept of symmetry from that of groups and Lie algebras to that of supergroups and super-Lie algebras. Then, a physically acceptable extension of the Poincaré Lie algebra exists: The super-Poincaré Lie algebra. The analysis of Coleman and Mandula was repeated by Haag, Lopuszański, and Sohnius for the super-Lie algebra case [4]. They found the super-Poincaré Lie algebra (in its versions with various numbers of supersymmetries and additional central charges) to be the only physically acceptable extension of the Poincaré Lie algebra.

Is this the end of the story? Can we go beyond supergroups and supersymmetry? And why "super" in the first place?

In the following we try to answer these questions from a categorial (or representation theoretic) point of view. This leads us to a unified view of symmetry and statistics through braided categories and quantum groups (in Section 2.1). This generalized notion of symmetry then provides the natural framework for posing the analogue of the extension problem (何) (in 
Section 2.2. In Section 3 we introduce the reader to the necessary essentials from quantum group theory and provide some elementary examples. Section 4.1 is devoted to reconstructing the quantum group symmetry underlying ordinary quantum field theory. As it turns out this is not the ordinary Poincaré group but a closely related quantum group. The reconstruction is then generalized (in Section 4.3) and applied to the extension problem (in Section 4.4). The latter section provides the link between the superextension problem and our generalized extension problem. In Section 5 we pursue the question of whether (for ordinary QFT) there is something "beyond supersymmetry". The answer is "No" and indeed the main mathematical result here is that all possible extensions (in the case of Bose-Fermi statistics) can be obtained from groups or supergroups.

As it turns out, our setting also provides us with new mathematical tools for dealing with supersymmetry. These are the tools of quantum geometry [5]. By quantum geometry we mean here the noncommutative geometry whose manifold-objects are algebras and whose group-objects are quantum groups (Hopf algebras). In quantum geometry there are generalizations of principal bundles, homogeneous spaces, differential forms etc. We give examples in Section 6 of how all this can be applied to supersymmetry and facilitates supersymmetric constructions. These include semidirect superextensions, the OSp-supergroups, super-spheres and the super-Poincaré group.

Proofs for mathematical statements are in general omitted as they are either known or straightforward. In the former case either a reference is given or they can be found in text books on quantum groups. An exception forms Theorem 5.1 whose proof is explicitly given.

We work throughout over the field of complex numbers.

\section{The Generalized Extension Problem}

\subsection{Why Quantum Group Symmetries?}

With the insufficiency of the group context in mind, we search for a more general but natural framework for the notion of symmetry and the extension problem. We are hereby guided by the categorial (i.e. representation theoretic) aspects of quantum field theory.

What are the "objects" that we deal with? States, fields, operators, Lagrangians etc. all live in vector spaces over $\mathbb{R}$ or $\mathbb{C}$. Furthermore, they all carry actions of the Poincaré group $\hat{P}$ or some larger symmetry group of the theory. That is, these vector spaces are representations of the symmetry group. Furthermore, there are maps between the representations which are required to be intertwiners, i.e., they commute with the group action. For example, an invariant operator can be viewed as such a map between states. What we have described so far, objects and maps between them, 
is essentially what makes a category. In this case, it is the category of representations of the symmetry group.

An essential operation in quantum field theory is the formation of tensor products of representations, e.g., to form a two-particle state out of two one particle states. This gives additional structure to the category of representations of the symmetry group and makes it into a monoidal category. In fact, this monoidal category carries all the information about the representation theory and we can forget about the group itself altogether.

We already know that we need to generalize the symmetry concept beyond that of groups to allow for supersymmetry. However, replacing groups by supergroups leads to monoidal categories as well. Conversely, given a monoidal category we require no knowledge about an underlying group or supergroup to perform all the representation theoretic operations necessary in quantum field theory. Thus, it appears natural to define a generalized concept of symmetry simply by that of a monoidal category.

However, there is a theorem of quantum group theory that states that for any monoidal category (with duals) there is a Hopf algebra so that the monoidal category is its category of representations.? This is called Tannaka-Krein reconstruction (see [5]). In fact, this gives rise to a one-toone correspondence between monoidal categories and Hopf algebras. Thus, the abstract generalization to any monoidal category gives us back a more concrete object that encodes the symmetries - a Hopf algebra. In the group case, this Hopf algebra is the commutative Hopf algebra of functions on the group.2 In the supergroup case the relation to the corresponding Hopf algebra is slightly more complicated (see Section (4).

We can go on to exploit our categorial point of view further to encompass the notion of particle statistics as well. In fact, this turns out to be essential as symmetry and statistics become inseparably linked in the generalized Hopf algebraic context.

A bit less obvious, it is also an essential ingredient of quantum field theory to have for two representations $V$ and $W$ an intertwiner $V \otimes W \rightarrow$ $W \otimes V$. For two one-particle states this intertwiner tells us what the exchange statistics of the particles is. E.g., for Bosons this would be $v \otimes w \mapsto w \otimes v$ while for Fermions we would have an extra minus sign $v \otimes w \mapsto-w \otimes v$. In general, the definition of such an intertwiner for any pair of representations is called a braiding. Thus, the objects of a quantum field theory live in a braided monoidal category. This encodes now both, the symmetries and the statistics of the theory. Note that this concept allows for more general

\footnotetext{
${ }^{1}$ We use the word "representation" for a Hopf algebra here and in the following to mean "comodule". See Section 3 for more details.

${ }^{2} \mathrm{We}$ are somewhat sloppy here and in the following concerning functional analytic questions such as the choice of class of functions on a space or the necessity to complete tensor products, consider multiplier algebras etc. The treatment of these questions would unnecessarily complicate the discussion and is irrelevant for the purposes of this paper.
} 
statistics than Bose and Fermi, see [6] for a discussion.

The braiding on the category as a category of representations yields an extra structure on the corresponding Hopf algebra via Tannaka-Krein reconstruction. This is called a coquasitriangular structure. Again, this gives rise to a one-to-one correspondence between braided monoidal categories and coquasitriangular Hopf algebras. In the following, we use the term quantum group to denote coquasitriangular Hopf algebras.

Importantly, it is not possible to combine arbitrary Hopf algebras with arbitrary braidings. To the contrary, for a given Hopf algebra the set of possible braidings on its representation category (encoded in the coquasitriangular structure) is usually very limited. Thus, symmetry and statistics cannot be viewed as separate entities in general. We subsume both under a generalized notion of symmetry which replaces ordinary groups by quantum groups. Unsurprisingly, also supersymmetry gives rise to a particular example of such a generalized symmetry, as we shall discuss in Section 4.4 .

\subsection{The Quantum Group Extension Problem}

Let us examine the extension problem (1) from the same abstract representation theoretic point of view that we have employed in the previous section.

Suppose we wish to embed a group $G$ into a larger group $G^{\prime}$. That is, we look for an inclusion $G \hookrightarrow G^{\prime}$. For the moment suppose we are just given a group homomorphism $G \rightarrow G^{\prime}$. For the representations this means that we can pull back a representation of $G^{\prime}$ to one of $G$. In fact, this gives rise to a (monoidal) functor between the (monoidal) categories of representations of the groups in the opposite direction $G_{G^{\prime}} \mathcal{M} \rightarrow{ }_{G} \mathcal{M}$. That is, for every representation of $G^{\prime}$ we get one of $G$ and for every intertwiner between representations of $G^{\prime}$ we get one between representations of $G$. Conversely, given this functor we can reconstruct the group homomorphism. Indeed, there is a one-to-one correspondence between such functors and group homomorphisms.

Generalizing as in the previous section to the case of arbitrary monoidal categories we still have such a correspondence. It is between functors and Hopf algebra homomorphisms. This time, both arrows point in the same direction. Thus, the generalization of the group homomorphism $G \rightarrow G^{\prime}$ is a Hopf algebra homomorphism $H^{\prime} \rightarrow H$. We recover the group case from the Hopf algebra case with the function Hopf algebras $H=\mathcal{C}(G), H^{\prime}=\mathcal{C}\left(G^{\prime}\right)$. The injectivity of the group homomorphism corresponds to the surjectivity of the Hopf algebra homomorphism. Thus, the problem of finding a "larger" group $G^{\prime}$ in which to embed a group $G$ generalizes to the problem of finding a "larger" Hopf algebra $H^{\prime}$ with a surjection $H^{\prime} \rightarrow H$ to the given Hopf algebra $H$.

While in the group extension problem (1) the exchange statistics is not 
explicitly mentioned and only enters separately in the physical conditions we can do better with our generalized setting of Section 2.1. To include the statistics we only have to consider the braiding that encodes it as well. Thus, we have braided monoidal categories instead of just monoidal categories. For a (monoidal) functor between such categories we impose the natural condition of being braided, i.e., of commuting with the braiding. This exactly expresses the condition that the statistics is preserved by the extension. We then have a correspondence between braided monoidal functors and homomorphisms of coquasitriangular Hopf algebras (quantum groups). Thus, the extension problem becomes that of finding a "larger" quantum group $H^{\prime}$ with a surjection (of coquasitriangular Hopf algebras) $H^{\prime} \rightarrow H$ to a given quantum group.

The analogue of the condition that the "larger" group not be a direct product corresponds to the "larger" quantum group not being a tensor product. Thus, we can formulate the quantum group generalization of (1) as follows: Denoting the relevant quantum group version of the Poincaré group by $\hat{P}^{\prime}$, find a quantum group $S \hat{P}^{\prime}$ and a surjection

$$
S \hat{P}^{\prime} \rightarrow \hat{P}^{\prime} \quad \text { such that } \quad S \hat{P}^{\prime} \neq \hat{P}^{\prime} \otimes G
$$

for any quantum group $\mathrm{G}$.

\section{Essentials from Quantum Group Theory}

In this section we introduce a few essential elements of quantum group theory and give some elementary examples. The latter serve to acquaint the reader with the formalism and form at the same time the basis for supersymmetric examples in Section 6. Most of the material in this section is text book knowledge. A good reference is Majid's book [5], in particular for the braided aspects. For the material on specific groups and Lie algebras see e.g. [7].

We assume the reader to be familiar with the notions of Hopf algebra, module, comodule, and Hopf algebra pairing. We use the notations $\Delta, \epsilon, \mathrm{S}$ for coproduct, counit and antipode of a Hopf algebra. We use Sweedler's notation (with implicit summation) $\Delta a=a_{(1)} \otimes a_{(2)}$ for coproducts and a similar notation $v \mapsto v_{(1)} \otimes v_{(\underline{2})}$ for left coactions.

A braided monoidal category is a monoidal category (i.e. a collection of objects and maps with a tensor product and certain compatibility conditions) so that for any two objects $V, W$ there is an invertible map $\psi$ : $V \otimes W \rightarrow W \otimes V$ (the braiding). The collection of $\psi$ 's also has to satisfy certain compatibility conditions. A braiding is called symmetric if $\psi=\psi^{-1}$.

\footnotetext{
${ }^{3} \hat{\mathrm{P}}^{\prime}$ encodes now the Poincaré symmetry as well as Bose-Fermi statistics and the spinstatistics relation. It is derived in Section 4.1.
} 
A coquasitriangular structure $\mathcal{R}: H \otimes H \rightarrow \mathbb{C}$ on a Hopf algebra $H$ provides a braiding on its category of left comodules via

$$
\psi(v \otimes w)=\mathcal{R}\left(w_{(1)} \otimes v_{(1)}\right) w_{(\underline{2})} \otimes v_{(\underline{2})} .
$$

If $\mathcal{R}\left(a_{(1)} \otimes b_{(1)}\right) \mathcal{R}\left(b_{(2)} \otimes a_{(2)}\right)=\epsilon(a) \epsilon(b)$, then $\mathcal{R}$ is called cotriangular and the induced braiding is symmetric.

Dually, a quasitriangular structure $\mathrm{R} \in H \otimes H$ on a Hopf algebra $H$ provides a braiding on its category of left modules via

$$
\psi(v \otimes w)=\mathrm{R}_{2} \triangleright w \otimes \mathrm{R}_{1} \triangleright v
$$

with $\mathrm{R}_{1} \otimes \mathrm{R}_{2}:=\mathrm{R}$ (summation implied). If $\mathrm{R}^{-1}=\mathrm{R}_{2} \otimes \mathrm{R}_{1}$, then $\mathrm{R}$ is called triangular and the induced braiding is symmetric.

As alluded to above, a group $G$ gives rise to a Hopf algebra as follows. Take the algebra of functions $\mathcal{C}(G)$ on $G$ and equip it with a coproduct defined by $(\Delta f)(g, h)=f(g h)$ for $f \in \mathcal{C}(G)$ and $g, h \in G$ using the identification $\mathcal{C}(G \times G) \cong \mathcal{C}(G) \otimes \mathcal{C}(G)$. Counit and antipode are given by $\epsilon(f)=f(e)$ and $(\mathrm{S} f)(g)=f\left(g^{-1}\right)$ where $e$ denotes the unit element of the group. Note that the Hopf algebra $\mathcal{C}(G)$ naturally carries the trivial cotriangular structure $\mathcal{R}=\epsilon \otimes \epsilon$ which encodes the trivial braiding $v \otimes w \mapsto w \otimes v$.

For matrix groups the corresponding Hopf algebra can be constructed rather explicitly. Consider the coalgebra with basis $\left\{t_{i j}\right\}$ for $i, j \in\{1, \ldots, n\}$, with coproduct $\Delta t_{i j}=\sum_{k} t_{i k} \otimes t_{k j}$ and counit $\epsilon\left(t_{i j}\right)=\delta_{i j}$. It is called the $n$-dimensional matrix coalgebra and is dual to the algebra of $n \times n$-matrices $M_{n}$. The free commutative bialgebra generated by the $t_{i j}$ is the "prototype" of the function Hopf algebra of a matrix group. More precisely, a matrix group that is a subalgebra of $M_{n}$ determined by polynomial constraints corresponds to a Hopf algebra which is a quotient of the described bialgebra by relations corresponding to the constraints.

From here on we adopt the convention that we denote the Hopf algebra of functions on a group $G$ by $G$. The class of functions we usually choose are the representative functions. These are the functions that arise as matrix elements of finite-dimensional representations. Furthermore we sometimes consider a conjugation in this context. This is nothing but ordinary complex conjugation.

Example 3.1. Consider the group SU(2). Its Hopf algebra $\mathrm{SU}(2)$ of representative functions is generated by the matrix coalgebra $\left\{t_{i j}\right\}$ with $i, j \in$ $\left\{\frac{1}{2},-\frac{1}{2}\right\}$ and relation (with the notation \pm for $\pm \frac{1}{2}$ )

$$
t_{--} t_{++}-t_{-+} t_{+-}=1 \text {. }
$$

$\mathrm{SU}(2)$ has a (Peter-Weyl) basis $\left\{t_{i j}^{l}\right\}$ with $l \in \frac{1}{2} \mathbb{N}_{0}$ and $i, j \in\{-l,-l+1, \ldots l\}$ with $t_{00}^{0}=1$ and $t_{i j}^{1 / 2}=t_{i j}$. Coproduct, counit, antipode and conjugation in 
this basis are given by

$$
\Delta t_{m n}^{l}=\sum_{k} t_{m k}^{l} \otimes t_{k n}^{l}, \quad \epsilon\left(t_{m n}^{l}\right)=\delta_{m n}, \quad \overline{t_{m n}^{l}}=\mathrm{S} t_{n m}^{l}=(-1)^{n-m} t_{-m,-n}^{l} .
$$

Example 3.2. Consider the group $\operatorname{Spin}(3,1)=S L(2, \mathbb{C})$ which is the double cover of the Lorentz group $S O(3,1)$. Its Hopf algebra Spin $(3,1)$ of representative functions is the tensor product of two copies of $\mathrm{SU}(2)$ whose generators we denote by $\left\{t_{i j}\right\}$ and $\left\{\overline{t_{i j}}\right\}$. However its conjugation is different, as indicated by the notation for the generators. A Peter-Weyl basis is thus given by $\left\{t_{i j}^{l}, \overline{t_{i j}^{l}}\right\}$.

Let $\left\{\sigma^{\mu}\right\}$ denote the standard Pauli matrices

$$
\sigma^{0}:=\left(\begin{array}{ll}
1 & 0 \\
0 & 1
\end{array}\right), \quad \sigma^{1}:=\left(\begin{array}{cc}
0 & 1 \\
1 & 0
\end{array}\right), \quad \sigma^{2}:=\left(\begin{array}{cc}
0 & -\mathrm{i} \\
\mathrm{i} & 0
\end{array}\right), \quad \sigma^{3}:=\left(\begin{array}{cc}
1 & 0 \\
0 & -1
\end{array}\right) .
$$

Define the $2 \times 2$ matrix $T$ of generators by

$$
T:=\left(\begin{array}{ll}
t_{--} & t_{-+} \\
t_{+-} & t_{++}
\end{array}\right)
$$

and the elements

$$
\Lambda^{\mu \nu}:=\frac{1}{2} \operatorname{tr}\left(\sigma^{\mu} T \sigma^{\nu} T^{\dagger}\right)
$$

where $T^{\dagger}$ is transposition of the matrix composed with conjugation of its elements. $\left\{\Lambda^{\mu \nu}\right\}$ generates precisely the sub-Hopf algebra $\mathrm{SO}(3,1)$ of functions on the Lorentz group. Note that $\overline{\Lambda^{\mu \nu}}=\Lambda^{\mu \nu}$.

The surjection $\operatorname{Spin}(3,1) \rightarrow \mathrm{SU}(2)$ corresponding to the injection $S U(2) \hookrightarrow$ $\operatorname{Spin}(3,1)$ is simply given by $t_{i j}^{l} \mapsto t_{i j}^{l}$ and $\overline{t_{i j}^{l}} \mapsto \overline{t_{i j}^{l}}$. (Note the different meaning of the conjugation in source and target.)

Not only a Lie group, but also a Lie algebra g gives rise to a Hopf algebra. More precisely, its universal envelope $\mathcal{U}(\mathfrak{g})$ can be made into a Hopf algebra. This is achieved by equipping the Lie algebra generators with the primitive coproduct $\Delta \eta=\eta \otimes 1+1 \otimes \eta$. This determines a coproduct on the whole of $\mathcal{U}(\mathfrak{g})$. The counit is given by $\epsilon(\eta)=0$ on the generators and the antipode by $\mathrm{S} \eta=-\eta$. Note that $\mathcal{U}(\mathfrak{g})$ is cocommutative. Furthermore, we sometimes consider a conjugation. Then the Lie algebra can be considered as the complexification of a real Lie algebra with the given complex conjugation.

It is a remarkable fact of Hopf algebra theory that the Hopf algebras obtained from a Lie group and its Lie algebra are dual to each other.

\footnotetext{
${ }^{4}$ The term "Peter-Weyl basis" refers here (as above) to a decomposition in terms of irreducible finite-dimensional representations and does not involve unitarity in any way.
} 
Example 3.3. Consider the Lie algebra $\mathfrak{s u}_{2}$ with basis $E, F, H$ and relations $[H, E]=2 E,[H, F]=-2 F,[E, F]=H$. Conjugation is given by $\bar{H}=-H$, $\bar{E}=-F, \bar{F}=-E$. Its universal enveloping Hopf algebra $\mathcal{U}\left(\mathfrak{s u}_{2}\right)$ is dually paired with $\mathrm{SU}(2)$ via

$$
\begin{aligned}
&\left\langle H, t_{m n}^{l}\right\rangle= 2 n \delta_{m, n}, \quad\left\langle E, t_{m n}^{l}\right\rangle=\sqrt{(l-n)(l+n+1)} \delta_{m, n+1} \\
&\left\langle F, t_{m n}^{l}\right\rangle=\sqrt{(l+n)(l-n+1)} \delta_{m, n-1}
\end{aligned}
$$

Example 3.4. Consider the Lie algebra $\mathfrak{s o}_{3,1}$ with basis $E, F, H, \bar{E}, \bar{F}, \bar{H}$. Apart from the different conjugation (indicated in the basis) it has the relations of $\mathfrak{s u}_{2} \oplus \mathfrak{s u}_{2}$ in the obvious way. The pairing of $\mathcal{U}\left(\mathfrak{s o}_{3,1}\right)$ with $\operatorname{Spin}(3,1)$ is as in Example 3.马 for the un-barred and the same for the barred generators. The pairing between un-barred and barred generators is zero.

For the elements $\Lambda^{\mu \nu}$ the pairing comes out as

$$
\begin{aligned}
& \left\langle X, \Lambda^{\mu \nu}\right\rangle=\frac{1}{2} \operatorname{tr}\left(\sigma^{\mu} \sigma(X) \sigma^{\nu}\right), \quad\left\langle\bar{X}, \Lambda^{\mu \nu}\right\rangle=\overline{\left\langle X, \Lambda^{\mu \nu}\right\rangle} \quad \forall X \in\{H, E, F\} \\
& \text { where } \sigma(H):=-\sigma^{3}, \quad \sigma(E):=\frac{1}{2}\left(\sigma^{1}-\mathrm{i} \sigma^{2}\right), \quad \sigma(F):=\frac{1}{2}\left(\sigma^{1}+\mathrm{i} \sigma^{2}\right)
\end{aligned}
$$

The injection $\mathfrak{s u}_{2} \hookrightarrow \mathfrak{s o}_{3,1}$ corresponding by duality to the surjection of Example 3.9 is given by $E \mapsto E-\bar{F}, F \mapsto F-\bar{E}, H \mapsto H-\bar{H}$. It extends to $\mathcal{U}\left(\mathfrak{s u}_{2}\right) \hookrightarrow \mathcal{U}\left(\mathfrak{s o}_{3,1}\right)$.

The simplest example of a Hopf algebra with non-trivial cotriangular structure (i.e. implying non-trivial braiding) is the following one.

Example 3.5. Let $\mathrm{Z}_{2}^{\prime}$ be the Hopf algebra of functions on $\mathbb{Z}_{2}$. It has two elements $1, g$ with relation $g^{2}=1$, coproduct $\Delta g=g \otimes g$, counit $\epsilon(g)=1$, and antipode $\mathrm{S} g=g$. We equip it with the cotriangular structure determined by $\mathcal{R}(g \otimes g)=-1$.

$Z_{2}^{\prime}$ is precisely the quantum group that generates the category of $\mathbb{Z}_{2^{-}}$ graded vector spaces as its category of comodules. A comodule $V$ of $\mathrm{Z}_{2}{ }^{\prime}$ splits into a direct sum $V_{0} \oplus V_{1}$ of even and odd part determined by the coaction $v \mapsto g^{|v|} \otimes v$. This is the natural setting for supergroups and superLie algebras. We start with more general definitions.

A braided Hopf algebra is the analogue of a Hopf algebra in a braided category. That is, it obeys the same axioms as an ordinary Hopf algebra except for the axiom of compatibility between product and coproduct which is modified to

$$
\Delta \circ \cdot=(\cdot \otimes \cdot) \circ(\mathrm{id} \otimes \psi \otimes \mathrm{id}) \circ(\Delta \otimes \Delta) .
$$


Definition 3.6. Let $A$ be an algebra in a braided category. $A$ is called braided commutative if $\cdot=\cdot \circ \psi$ is an identity of maps $A \otimes A \rightarrow A$.

Dually, let $C$ be a coalgebra in a braided category. $C$ is called braided cocommutative if $\Delta=\psi \circ \Delta$ is an identity of maps $C \rightarrow C \otimes C$.

Now, in the same way as ordinary Hopf algebras describe groups and enveloping algebras, $\mathbb{Z}_{2}$-graded Hopf algebras (i.e., braided Hopf algebras in the category of $Z_{2}{ }^{\prime}$-comodules) describe supergroups and super-Lie algebras. Thus supergroups are described by $\mathbb{Z}_{2}$-graded commutative Hopf algebras and super-enveloping algebras by $\mathbb{Z}_{2}$-graded cocommutative Hopf algebras. The latter case is more familiar. One usually considers super-Lie algebras. Those give indeed rise to enveloping Hopf algebras precisely in the same way as ordinary Lie algebras do, except that everything takes place in the $\mathbb{Z}_{2}$-graded category. Our definition of supergroups might seem less familiar but is standard in the quantum groups literature (see e.g. [8] where even $q$-deformations of supergroups were considered). It is also much less complicated than analytically inspired definitions using auxiliary Grassmann algebras.

In fact, one can generalize these considerations to arbitrary braiding employing the notion of braided Hopf algebra mentioned above, see [5]. However, we shall limit ourselves mostly to the $\mathbb{Z}_{2}$-graded case, occasionally generalizing to arbitrary symmetric braidings. For non-symmetric braidings additional problems occur, most notably the absence of an analogue of Proposition 3.10 (as discussed in Section 4.3).

We are now ready to define the extension problems more precisely (disregarding for now the requirement that the extension must not be a direct product respectively tensor product). The conventional version (1), generalized to encompass e.g. supergroups in the abovementioned sense can be formulated as follows:

Definition 3.7. Let $H$ be a braided commutative Hopf algebra in a symmetric braided category. Then, the triangular group extension problem is the problem to find a braided commutative Hopf algebra $B$ in the category with a surjection $\sigma: B \rightarrow H$. Any such $B$ is said to be a solution of the problem.

In the ordinary group case the underlying category is just the category of vector spaces and the braiding is simply the interchange of the tensor components. $H$ is thus an ordinary commutative Hopf algebra which encodes the algebra of functions on a group. In the supergroup case the category is that of $\mathbb{Z}_{2}$-graded vector spaces and the braiding is the interchange with an additional minus sign if both components are odd. Thus, $H$ is a graded commutative Hopf algebra which encodes the algebra of functions on a supergroup.

The quantum group extension problem (2) takes the following form: 
Definition 3.8. Let $H$ be a coquasitriangular Hopf algebra. Then, the quantum group extension problem is the problem to find a coquasitriangular Hopf algebra $A$ with a surjection $\pi: A \rightarrow H$. Any such $A$ is said to be a solution of the problem.

For the following discussion of reconstruction we require the analogue of a semidirect product of groups for Hopf algebras. This is provided by the following theorem and its variants. Their significance will become clear in the next section.

Theorem 3.9 (Majid [9, 10]). Let $H$ be a coquasitriangular Hopf algebra, $B$ a braided Hopf algebra in the braided category ${ }^{H} \mathcal{M}$ of left $H$-comodules. Then, there exists a Hopf algebra $B \rtimes H$, called the bosonization of $B$, such that the category of left comodules of $B \rtimes H$ is monoidal equivalent to the category of braided left comodules of $B$ in ${ }^{H} \mathcal{M}$.

Explicitly, $B \rtimes H$ is isomorphic to $B \otimes H$ as a vector space. Its product, coproduct, and antipode are given by

$$
\begin{gathered}
(b \otimes h)(c \otimes g)=\mathcal{R}\left(c_{[1]} \otimes h_{(1)}\right) b c_{[2]} \otimes h_{(2)} g \\
\Delta(b \otimes h)=\left(b_{(1)} \otimes b_{(2)[1]} h_{(1)}\right) \otimes\left(b_{(2)[2]} \otimes h_{(2)}\right) \\
\mathrm{S}(b \otimes h)=\mathcal{R}\left(\left(\mathrm{S}_{B} b_{[2]}\right)_{[1]} \otimes\left(\mathrm{S}_{H}\left(b_{[1]} h\right)\right)_{(1)}\right)\left(\mathrm{S}_{B} b_{[2]}\right)_{[2]} \otimes\left(\mathrm{S}_{H}\left(b_{[1]} h\right)\right)_{(2)}
\end{gathered}
$$

for $b, c \in B$ and $h, g \in H$. Here, the coaction of $H$ on $B$ is denoted by $b \mapsto b_{[1]} \otimes b_{[\underline{2]}}$.

Furthermore, there is a Hopf algebra surjection $\pi: B \rtimes H \rightarrow H$ defined by $b \otimes h \mapsto \epsilon(b) h$ and an injection $i: H \hookrightarrow B \rtimes H$ defined by $h \mapsto 1 \otimes h$ such that $\pi \circ i=\mathrm{id}$. Conversely, let $\pi: A \rightarrow H$ be a Hopf algebra surjection. Then $A$ is a bosonization $A=B \rtimes H$ for some $B$ if and only if there is an injection $i: H \hookrightarrow A$ such that $\pi \circ i=\mathrm{id}$.

Proposition 3.10. In the context of Theorem 3.9 assume that $H$ is cotriangular and that $B$ is braided commutative. Then, $B \rtimes H$ inherits a cotriangular structure from $H$ by pull-back, i.e.,

$$
\mathcal{R}((b \otimes h) \otimes(c \otimes g)):=\mathcal{R}_{H}(h \otimes g) \epsilon(b) \epsilon(c) .
$$

Furthermore the equivalence of categories of Theorem 3.9 becomes an equivalence of braided categories in this way. In particular, $B \rtimes H \rightarrow H$ is a quantum group extension in the sense of Definition 3.8.

For the pairing of bosonizations we need the following lemma.

Lemma 3.11. Let $A$ be a coquasitriangular Hopf algebra and $H$ a quasitriangular Hopf algebra which are dually paired via $H \otimes A \rightarrow \mathbb{C}$. Let $B$ be an $A$-comodule braided Hopf algebra and $D$ an $H$-module braided Hopf algebra 
such that they are dually paired as algebra/coalgebra and coalgebra/algebra. . Furthermore, we demand the compatibility condition of action and coaction

$$
\langle h \triangleright d, b\rangle=\left\langle h, b_{(1)}\right\rangle\left\langle d, b_{(\underline{2})}\right\rangle \quad \forall h \in H, b \in B, d \in D .
$$

Then the bosonizations $D \rtimes H$ and $B \rtimes A$ are naturally dually paired via

$$
\langle d \otimes h, b \otimes a\rangle:=\langle d, b\rangle\langle h, a\rangle \quad \forall h \in H, a \in A, b \in B, d \in D .
$$

As an example of how the bosonization construction reduces to an ordinary semidirect product for groups and Lie algebras we consider the Poincaré group and its Lie and (enveloping) algebra as well as their pairing.

Example 3.12. In the context of Example 3.9 consider the Hopf algebra Mink of (polynomial) functions on the translation group of Minkowski space. It is generated by $\left\{x^{\mu}\right\}$ as a free commutative algebra with coproduct, antipode and conjugation

$$
\Delta x^{\mu}=x^{\mu} \otimes 1+1 \otimes x^{\mu}, \quad \mathrm{S} x^{\mu}=-x^{\mu}, \quad \overline{x^{\mu}}=x^{\mu} .
$$

It is a (trivially braided) Spin(3,1)-comodule Hopf algebra via the coaction $x^{\mu} \mapsto \sum_{\nu} \Lambda^{\mu \nu} \otimes x^{\nu}$.

The Hopf algebra of functions Poinc on the covered Poincaré group is the bosonization Mink $\rtimes \mathrm{Spin}(3,1)$. As an algebra it is the commutative algebra generated by Spin $(3,1)$ and Mink. The coalgebra structure and antipode for $t_{i j}$ is that of $\operatorname{Spin}(3,1)$. The coproduct and antipode for $x^{\mu}$ are given by

$$
\Delta x^{\mu}=x^{\mu} \otimes 1+\sum_{\nu} \Lambda^{\mu \nu} \otimes x^{\nu}, \quad \mathrm{S} x^{\mu}=-\sum_{\nu}\left(\mathrm{S} \Lambda^{\mu \nu}\right) x^{\nu} .
$$

Note that the sub-Hopf algebra generated by $\left\{x^{\mu}, \Lambda^{\mu \nu}\right\}$ is the uncovered Poincaré group Mink $\rtimes \mathrm{SO}(3,1)$.

Example 3.13. In the context of Example 3.4 let $\mathfrak{t r}_{4}$ denote the abelian Lie algebra of translation generators in 4 dimensions with basis $\left\{P^{\mu}\right\}$ and real structure $\overline{P^{\mu}}=P^{\mu}$. Its universal enveloping Hopf algebra $\mathcal{U}\left(\mathfrak{t r}_{4}\right)$ is a (trivially braided) $\mathcal{U}\left(\mathfrak{s o}_{3,1}\right)$-module Hopf algebra by the action

$$
X \triangleright P^{\mu}=\frac{1}{2} \sum_{\nu} \operatorname{tr}\left(\sigma^{\nu} \sigma(X) \sigma^{\mu}\right) P^{\nu}, \quad \bar{X} \triangleright P^{\mu}=\overline{X \triangleright P^{\mu}} \quad \forall X \in\{H, E, F\} .
$$

The semidirect product of the Lie algebras is the Poincaré Lie algebra. Correspondingly for the enveloping Hopf algebras $\mathcal{U}(\mathfrak{p o i n c})=\mathcal{U}\left(\mathfrak{t r}_{4} \rtimes \mathfrak{s o}_{3,1}\right)=$ $\mathcal{U}\left(\mathfrak{t r}_{4}\right) \rtimes \mathcal{U}\left(\mathfrak{s o}_{3,1}\right)$.

$\mathcal{U}\left(\mathfrak{t r}_{4}\right)$ and Mink are dually paired via $\left\langle P^{\mu}, x^{\nu}\right\rangle=\delta^{\mu \nu}$. As this pairing is compatible with the action of $\mathcal{U}\left(\mathfrak{s o}_{3,1}\right)$ and coaction of $\operatorname{Spin}(3,1)$ in the sense of Lemma 3.11 it induces a pairing between the Hopf algebras $\mathcal{U}($ poinc) and Poinc.

\footnotetext{
${ }^{5}$ Note that we take the ordinary pairing here and not the type of pairing usually employed in braided categories. In particular, note that $B$ and $D$ do not live in the same braided category.
} 


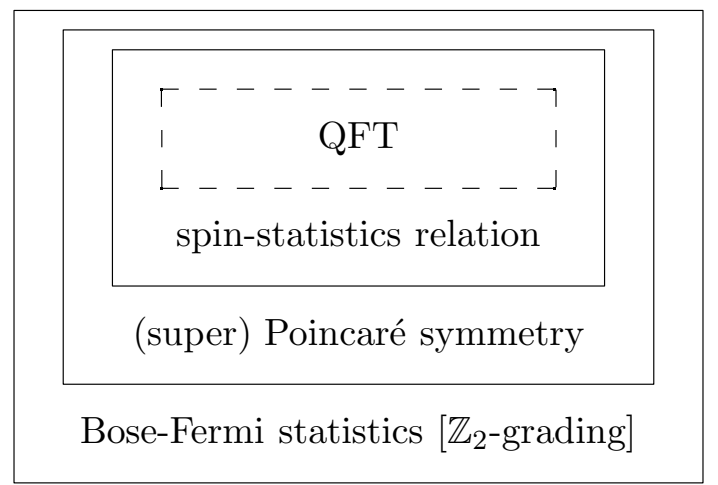

Figure 1: The three layers of the reconstruction of the quantum group symmetry. They correspond to successive restrictions of categories.

\section{Reconstruction of Quantum Group Symmetry}

\subsection{Poincaré Symmetry and Bose-Fermi statistics}

Let us reconstruct the relevant braided monoidal category (and quantum group) for a quantum field theory that is Poincaré symmetric, has BoseFermi statistics, and obeys the spin-statistics theorem. In fact, we simplify the discussion slightly by only considering the $S U(2)$ subgroup of $\hat{P}$ since it exhibits already all the relevant features. We come back to the full Poincaré group at the end. The construction proceeds in three "layers" corresponding respectively to the statistics, the symmetry group, and the spin-statistics relation, see Figure 1 .

The first layer (the outermost box in Figure 1) is an underlying $\mathbb{Z}_{2^{-}}$ grading. More precisely, we consider the braided category of $\mathbb{Z}_{2}$-graded vector spaces. This distinguishes Fermions from Bosons and carries the Bose-Fermi statistics. Reconstructing the quantum group that generates the category, we obtain the function Hopf algebra $Z_{2}^{\prime}$ of the group $\mathbb{Z}_{2}$, but with the nontrivial cotriangular structure, described in Example 3.5. The cotriangular structure provides the braiding

$$
\psi(v \otimes w)=(-1)^{|v| \cdot|w|} w \otimes v
$$

which encodes Bose-Fermi statistics.

The second layer (the intermediate solid box in Figure 1) is the symmetry group, in this case $S U(2)$. We wish to restrict our category of $\mathbb{Z}_{2}$-graded vector spaces to those spaces that are representations of the group $S U(2)$ as well. Furthermore, we require the group action to respect the grading. This means that we can view $S U(2)$ as living in the $\mathbb{Z}_{2}$-graded category itself. More precisely, taking the quantum group point of view, the corresponding function Hopf algebra $S U(2)$ is an object in the category. It is purely even 
under the $\mathbb{Z}_{2}$-grading. The braided category which encodes both the BoseFermi statistics as well as the $S U(2)$-symmetry is then the subcategory of comodules of $\mathrm{SU}(2)$ inside the category of $\mathbb{Z}_{2}$-graded vector spaces. However, according to the reconstruction theorem we can express the braided category as a category of representations of just one quantum group. We are here precisely in the situation of Theorem 3.9 and Proposition 3.10 which tell us that this quantum group is obtained from $\mathrm{Z}_{2}{ }^{\prime}$ and $\mathrm{SU}(2)$ by a kind of semidirect product, called bosonization $\mathrm{SU}(2) \rtimes \mathrm{Z}_{2}{ }^{\prime}$. In the case at hand this reduces just to the ordinary tensor product $S U(2) \otimes Z_{2}{ }^{\prime}$ since $S U(2)$ is purely even, i.e., trivial as a representation of $Z_{2}{ }^{\prime}$. Using the basis of $S U(2)$ given in Example 3.1 the tensor product $\mathrm{SU}(2) \otimes \mathrm{Z}_{2}{ }^{\prime}$ has a basis $\left\{t_{m n}^{l}, t_{m n}^{l} g\right\}$. Its coquasitriangular structure is given by

$$
\mathcal{R}\left(t_{m n}^{l} g^{k} \otimes t_{m^{\prime} n^{\prime}}^{l^{\prime}} g^{k^{\prime}}\right)=(-1)^{k k^{\prime}} \delta_{m n} \delta_{m^{\prime} n^{\prime}} .
$$

The third and final layer (the innermost solid box in Figure 1) consists of removing those representations that have the wrong spin-statistics relation. We only allow representations where either the spin-label is integer and the $\mathbb{Z}_{2}$-degree even or the spin-label non-integer and the $\mathbb{Z}_{2}$-degree odd. The coaction for a spin- $l$ representation thus must take the form

$$
v_{m} \mapsto \sum_{n} t_{m n}^{l} g^{2 l} \otimes v_{n}
$$

Allowing only representations of this form is equivalent to restricting the Hopf algebra $\mathrm{SU}(2) \otimes \mathrm{Z}_{2}^{\prime}$ to the sub-Hopf algebra spanned by $\left\{t_{m n}^{l} g^{2 l}\right\}$. More generally, restricting a monoidal category to a monoidal subcategory corresponds by Tannaka-Krein reconstruction exactly to restricting a Hopf algebra to a sub-Hopf algebra.

By renaming the element $t_{m n}^{l} g^{2 l}$ with $t_{m n}^{l}$ we recognize that the Hopf algebra we arrive at is nothing but $\mathrm{SU}(2)$ again. However, the coquasitriangular structure we obtain by restriction from $S U(2) \otimes Z_{2}^{\prime}$ is not the canonical (trivial) coquasitriangular structure of $\mathrm{SU}(2)$. Instead, it is given by

$$
\mathcal{R}\left(t_{m n}^{l} \otimes t_{m^{\prime} n^{\prime}}^{l^{\prime}}\right)=(-1)^{4 l l^{\prime}} \delta_{m n} \delta_{m^{\prime} n^{\prime}} .
$$

To make this distinction clear we denote the new coquasitriangular Hopf algebra by $\mathrm{SU}^{\prime}(2)$. It is precisely the one that was already found in [6] by considering spin and statistics symmetries directly.

The construction generalizes to double covers of space-time symmetry groups. Thus, assume a given space-time symmetry group $G$ whose universal cover (i.e., quantum mechanically relevant symmetry) is a double cover $\hat{G}$. Then, the function Hopf algebra decomposes into a direct sum $\hat{\mathrm{G}}=\hat{\mathrm{G}}_{s} \oplus \hat{\mathrm{G}}_{a}$ with $\hat{\mathrm{G}}_{s}=\mathrm{G}$ of functions that are symmetric respectively antisymmetric with respect to interchange of the sheets of the cover. As the direct sum is a direct sum of coalgebras this introduces a grading on the 
representations which corresponds precisely to spin (i.e., grading in integer versus half-integer spin). Assuming the usual spin-statistics relation we obtain as above $\hat{G}$ itself as the relevant symmetry quantum group but with the cotriangular structure of the following lemma. This generalizes (3). See also [6].

Lemma 4.1. Let $H$ be a commutative Hopf algebra which is $\mathbb{Z}_{2}$-graded as an algebra into a direct sum of subcoalgebras $H=H_{0} \oplus H_{1}$. Then

$$
\mathcal{R}(f \otimes g)=(-1)^{|f||g|} \epsilon(f) \epsilon(g),
$$

where $|f|$ is the degree of $f$ with respect to the grading, defines a cotriangular structure on $H$.

In particular, for the (covering) Lorentz group Spin $(3,1)$ and the full Poincaré group Poinc the grading is given by $\left|t_{i j}^{l}\right|=\left|\overline{t_{i j}^{l}}\right|=2 l(\bmod 2)$ and $\left|x^{\mu}\right|=0$. We denote the versions with the cotriangular structure (4) by $\operatorname{Spin}^{\prime}(3,1)$ and Poinc' respectively.

Remark 4.2. There did not seem to be any intrinsic reason to put the $\mathbb{Z}_{2-}$ grading encoding the Bose-Fermi statistics "below" the conventional symmetry group. The bosonization appearing above really is just an ordinary tensor product. However, when we go to supersymmetric groups such as the super-Poincaré group this is no longer true. In this case the reconstruction really requires the $\mathbb{Z}_{2}$-grading to lie "below" as now the group is non-trivially graded. See Sections 4.3 and 4.4.

\subsection{The Dual Context}

As it is more familiar to physicists we also describe the dual context with Lie algebras and universal enveloping algebras. Thus, let $\mathfrak{g}$ be the Lie group of a space-time symmetry group $G$ as considered above. Its universal enveloping Hopf algebra $\mathcal{U}(\mathfrak{g})$ is dually paired with the function Hopf algebra $G$ as well as with the function Hopf algebra of the double cover $\hat{G}$. We can describe comodules of $\hat{G}$ alternatively as modules of $\mathcal{U}(\mathfrak{g})$. However, the global information about the difference between $G$ and $\hat{G}$ that contains the information about (integer versus half-integer) spin is lost. But we can recover the information by adjoining an element $\xi$ to $\mathcal{U}(\mathfrak{g})$ with $\xi^{2}=1$ which commutes with all other elements and has coproduct $\Delta \xi=\xi \otimes \xi$. We extend the pairing with $\hat{G}$ by defining

$$
\langle\xi, f\rangle=(-1)^{|f|} \epsilon(f) \quad \forall f \in \hat{\mathrm{G}} .
$$

The action of $\xi$ on a representation should yield the eigenvalues $1,-1$ depending on whether spin is "integer" or "half-integer". To ensure this we need to formally identify $\xi$ with $(-1)^{C}$ where $C$ is a suitable operator having even/odd eigenvalues on "integer" / "half-integer" spin representations. 
(Note that given such an operator, $(-1)^{C}$ is automatically central, idempotent, and group-like.)

Following the construction above then leads to this version of $\mathcal{U}(\mathfrak{g})$ as the spin-statistics reduced (dual) quantum group with the non-trivial triangular structure given by the following lemma.

Lemma 4.3. Let $A$ be a cocommutative Hopf algebra with a central element $\xi$ satisfying $\xi^{2}=1$ and $\Delta \xi=\xi \otimes \xi$. Then it admits a triangular structure

$$
\mathrm{R}=\frac{1}{2}(1 \otimes 1+1 \otimes \xi+\xi \otimes 1-\xi \otimes \xi)
$$

Thus, we see that we can do everything in the dual enveloping algebra context as well, though at the price that the global structure needs to be added by hand. This is one reason why we prefer the function algebra setting.

In fact, we could have performed the reconstruction from the beginning in the enveloping algebra setting. Then, the element $\xi$ (corresponding to the element $g$ generating $C^{\prime}\left(\mathbb{Z}_{2}\right)$ ) would have come from the (dual) bosonization construction for the enveloping algebra. The final step of the spin-statistics reduction then precisely corresponds to identifying $\xi=(-1)^{C}$.

Example 4.4. In the context of Example 3.9 we adjoin the element $\xi$ to $\mathcal{U}\left(\mathfrak{s u}_{2}\right)$ as described above which we formally equate with $(-1)^{C}$ where $C:=$ $4 E F+2 H+H^{2}$. Since $\left\langle C, t_{m n}^{l}\right\rangle=4 l(l+1) \delta_{m, n}$ we get

$$
\left\langle\xi, t_{m n}^{l}\right\rangle=(-1)^{4 l(l+1)} \delta_{m, n}=(-1)^{2 l} \delta_{m, n}
$$

as required. We denote this version of the enveloping algebra with the triangular structure (河) by $\mathcal{U}^{\prime}\left(\mathfrak{s u}_{2}\right)$.

We proceed similarly for Example 3.4 and define $\mathcal{U}^{\prime}\left(\mathfrak{s o}_{3,1}\right)$ with the operator $C+\bar{C}$ where $C$ is as defined above and equate $\xi=(-1)^{C+\bar{C}}$.

We define the bosonization $\mathcal{U}^{\prime}($ poinc $)=\mathcal{U}\left(\mathfrak{t r}_{4}\right) \rtimes \mathcal{U}^{\prime}\left(\mathfrak{s o}_{3,1}\right)$ analogous to Example 3.15 where $\xi$ acts trivially on $P^{\mu}$.

\subsection{Formalized Reconstruction}

We now formalize and generalize the procedure of reconstructing the symmetry quantum group, exposing more clearly the role of the different layers. (This section is somewhat more technical and can be omitted by nonexperts.)

The first layer is as before the underlying statistics. We generalize it from a $\mathbb{Z}_{2}$-grading given by $\mathbf{Z}_{2}^{\prime}$ to an arbitrary cotriangular Hopf algebra $H$. That is, the statistics is now encoded by the category of (left) $H$-comodules ${ }^{H} \mathcal{M}$. Cotriangularity implies that the braiding is symmetric, i.e., the braiding and its inverse are identical. This is in fact the limit of validity of the traditional 
separation of spin and statistics: When the braiding is non-symmetric such a separation is no longer possible. This is essentially because Proposition 3.10 does not generalize to the coquasitriangular case. To put it differently: The bosonization $B \rtimes H$ admits an induced coquasitriangular structure from $H$ in general only if $H$ is cotriangular.

For the second layer, the symmetry, we require now a braided commutative Hopf algebra $B$ in ${ }^{H} \mathcal{M}$. This generalizes the concepts of group and supergroup to arbitrary braiding. Again, using Theorem 3.9 and Proposition 3.10, the quantum group that generates the braided category of representations of $B$ inside ${ }^{H} \mathcal{M}$ is the bosonization $B \rtimes H$.

For the third layer, a spin-statistics relation obviously requires that we have a "spin" that we can put in correspondence with the statistics. In the previous section that came from the group $S U(2)$. In fact, the only relevant part of it (integer or non-integer spin) is encoded in the subgroup $\mathbb{Z}_{2}$ of $S U(2)$. More generally, we need the same (quantum) group as the one encoding the statistics but now as a quotient ("subgroup" in group language) of $B$. In other words, we require a surjection of braided Hopf algebras $\sigma: B \rightarrow H$ in ${ }^{H} \mathcal{M}$, where $H$ is trivial as an $H$-comodule.

To impose now the spin-statistics relation we observe that the surjection $\sigma: B \rightarrow H$ gives rise to a surjection of cotriangular Hopf algebras

$$
\tilde{\sigma}: \tilde{B}:=B \rtimes H \rightarrow H \rtimes H
$$

upon bosonization. In fact, $H \rtimes H=H \otimes H$ since the coaction of $H$ on $H$ was taken to be trivial. For an arbitrary object $V$ in the category of $\tilde{B}$ comodules, spin and statistics are given by the induced coaction of $H \otimes H$. Denoting the coaction by $\beta: V \rightarrow \tilde{B} \otimes V$ this induced coaction is given by $\tilde{\beta}:=(\tilde{\sigma} \otimes \mathrm{id}) \circ \beta: V \rightarrow H \otimes H \otimes V$. To understand what it means to satisfy the spin-statistics relation let us think for a moment in the more familiar language of groups. Thus, let us think that (the dual of) $\tilde{\beta}$ defines an action of two copies of the spin-statistics group $G$ on $V$. Now, $V$ obeys the spinstatistics relation if it is in the same representation with respect to both copies of $G$. We can express this formally by saying that the action of $G \times G$ factors through the action of a single copy of $G$ by the group multiplication $G \times G \rightarrow G$. Translating this back to the quantum group language means that the image of $\tilde{\beta}$ must lie in $(\Delta H) \otimes V$ where $\Delta H$ is the image of $H$ in $H \otimes H$ under the coproduct. This is precisely ensured by restricting $\tilde{B}$ to the largest sub-Hopf algebra $A \subseteq \tilde{B}$ so that $\tilde{\sigma}(A) \subseteq \Delta H$. In fact, $A$ is not in general the preimage $\tilde{\sigma}^{-1}(\Delta H)$ as this is not necessarily a Hopf algebra. One can derive a stronger condition directly from the properties of a comodule $V$. In fact, it is not enough that $V$ satisfies the spin-statistics relation in the form $\tilde{\beta}(V) \subseteq(\Delta H) \otimes V$. But applying the coproduct several times (or alternatively the coproduct on $H \otimes H$ ) the corresponding condition must hold for any copy of $H \otimes H$ that appears in the image. This leads to 
the condition (id $\otimes \tilde{\sigma} \otimes$ id) $\circ \Delta^{2}(A) \subseteq A \otimes \Delta H \otimes A$ which defines a bialgebra. As we require a Hopf algebra we need to impose the even more restrictive condition (id $\otimes \tilde{\sigma} \otimes$ id) $\circ \Delta^{2}(A) \subseteq A \otimes(\Delta H \cap \tau(\Delta H)) \otimes A$ where $\tau$ is the transposition map. This corresponds to requiring for a module $V$ that also its dual satisfies the spin-statistics relation. We call $A$ the spin-statistics reduction of $\tilde{B}$ and formalize as follows:

Definition 4.5. Let $H$ be a cotriangular Hopf algebra. Let $\sigma: B \rightarrow H$ be a solution of the triangular group extension problem in ${ }^{H} \mathcal{M}$, where $H$ is equipped with the trivial comodule structure under itself. Consider the induced map $\tilde{\sigma}: \tilde{B}:=B \rtimes H \rightarrow H \rtimes H=H \otimes H$. Then $A \subseteq \tilde{B}$ defined as the subspace satisfying

$$
(\mathrm{id} \otimes \tilde{\sigma} \otimes \mathrm{id}) \circ \Delta^{2}(A) \subseteq A \otimes(\Delta H \cap \tau(\Delta H)) \otimes A
$$

is a sub-Hopf algebra called the spin-statistics reduction.

\subsection{Extensions}

We now turn to the question of whether and how a group extension in the conventional (or triangular) sense gives rise to a quantum group extension.

Let us consider the Bose-Fermi case first. Thus, we have a $\mathbb{Z}_{2}$-graded Hopf algebra $B$ (e.g., the ordinary Poincaré group which is just trivially graded) and a $\mathbb{Z}_{2}$-graded extension $C$ of it (e.g., the super-Poincaré group). That is, we have a $\mathbb{Z}_{2}$-graded group extension $C \rightarrow B$ in $\mathrm{Z}_{2}{ }^{\prime} \mathcal{M}$. In general, we have some cotriangular Hopf algebra $H$ in place of $\mathrm{Z}_{2}{ }^{\prime}$ and $\rho: C \rightarrow B$ is a triangular extension in the sense of Definition 3.7. On the other hand, both $B$ and $C$ are also solutions to the extension problem for the "spin" $H$ as a trivial comodule in ${ }^{H} \mathcal{M}$. Thus we have surjections $\sigma_{B}: B \rightarrow H$ and $\sigma_{C}: C \rightarrow H$ as well and $\sigma_{C}=\sigma_{B} \circ \rho$. The categorial equivalence of Proposition 3.10 lifts these to surjections of cotriangular Hopf algebras $C \rtimes H \rightarrow B \rtimes H \rightarrow H \rtimes H=H \otimes H$.

We now apply the spin-statistics reduction of Definition 4.5. Denote the reduced quantum groups by $B^{\prime}$ and $C^{\prime}$. The image of $C^{\prime}$ obviously satisfies the reduction condition itself and thus we have a map $C^{\prime} \rightarrow B^{\prime}$ as the restriction of $C \rtimes H \rightarrow B \rtimes H$. However, this map is not necessarily surjective. Thus, we do not necessarily obtain a solution of the quantum group extension problem, but something weaker. The triangular extension could behave "badly" with respect to the spin-statistics relation.

\section{$5 \quad$ Nothing beyond Supersymmetry}

In this section we perform in a sense the opposite operation to the reconstruction of Section 4.4. We show, for the case of Bose-Fermi statistics, that any solution of the quantum group extension problem can be induced from a 
solution of the triangular group extension problem. Returning to the initial question wether a unification of external and internal degrees of freedom beyond supersymmetry is possible, this implies a negative answer. More precisely, any extension of the symmetry quantum group of ordinary quantum field theory (in at least 3 spatial dimensions) can already be obtained through the known (supersymmetric) ones.

We first consider the special case where the quantum group to be extended is just the spin (and thus also statistics) generating one. This is our main theorem.

Theorem 5.1. Let $\pi: A \rightarrow \mathrm{Z}_{2}^{\prime}$ be a solution of the quantum group extension problem for $\mathbf{Z}_{2}{ }^{\prime}$. Then, there is a solution $\sigma: B \rightarrow \mathbf{Z}_{2}$ of the $\mathbb{Z}_{2}$-graded group extension problem in $\mathrm{Z}_{2}{ }^{\prime} \mathcal{M}$ where $\mathrm{Z}_{2}{ }^{\prime}$ coacts trivially on itself, such that $A$ is the spin-statistics reduction of $B \rtimes \mathrm{Z}_{2}^{\prime}$.

Proof. Define the space $\tilde{B}:=A \otimes \mathrm{Z}_{2}^{\prime}$ and a surjection $\tilde{\sigma}: \tilde{B} \rightarrow \mathrm{Z}_{2} \otimes \mathrm{Z}_{2}{ }^{\prime}$ given by $a \otimes h \mapsto \pi\left(a_{(1)}\right) \otimes \pi\left(a_{(2)}\right) h$. This gives rise to the sequence

$$
\tilde{B} \stackrel{\tilde{\sigma}}{\rightarrow} \mathrm{Z}_{2} \otimes \mathrm{Z}_{2}^{\prime} \stackrel{\epsilon \otimes \mathrm{id}}{\longrightarrow} \mathrm{Z}_{2}^{\prime} .
$$

We give $\tilde{B}$ the tensor product coproduct structure, the subalgebra structures of $A$ and $\mathrm{Z}_{2}{ }^{\prime}$ and the cross relations induced by the pull-back of the cotriangular structure of $Z_{2}{ }^{\prime}$. This makes (7) into a sequence of cotriangular Hopf algebras. (Note that the coquasitriangular structure on $Z_{2} \otimes Z_{2}{ }^{\prime}$ is thus trivial on the first component, hence the notation without the prime.) Now consider the injection of cotriangular Hopf algebras $i: \mathrm{Z}_{2}{ }^{\prime} \rightarrow \tilde{B}$ given by $h \mapsto 1 \otimes h$. The surjection $\tilde{B} \rightarrow \mathrm{Z}_{2}^{\prime}:(\epsilon \otimes \mathrm{id}) \circ \tilde{\sigma}$ inverts $i$. Thus, according to Theorem 3.9 there is a braided Hopf algebra $B$ in the category of left $\mathrm{Z}_{2}{ }^{\prime}$-comodules so that $\tilde{B}=B \rtimes \mathrm{Z}_{2}{ }^{\prime}$. We can recover $B$ (as an algebra) as $\mathrm{Z}_{2}^{\prime} \tilde{B}$ and observe that on this space the map $\tilde{\sigma}$ restricts to the subspace $Z_{2} \otimes 1$ of $Z_{2} \otimes Z_{2}{ }^{\prime}$. Identifying $Z_{2} \otimes 1$ as $\mathbf{Z}_{2}{ }^{\prime}\left(Z_{2} \otimes Z_{2}{ }^{\prime}\right)$ we obtain precisely a surjection $\sigma: B \rightarrow \mathbf{Z}_{2}$ as required.

It remains to show that $A$ is the spin-statistics reduction $A^{\prime}$ of $\tilde{B}$. For this observe that th condition (6) of Definition 4.5 implies (id $\otimes \tilde{\sigma} \otimes \mathrm{id}) \circ \Delta^{2}\left(A^{\prime}\right) \subseteq$ $\tilde{B} \otimes\left(\Delta \mathrm{Z}_{2}^{\prime}\right) \otimes \tilde{B}$. This in turn implies for an element $a \otimes 1+b \otimes g$ in $A^{\prime}$ that $\left(a_{(1)} \otimes 1\right) \otimes 1 \otimes\left(a_{(2)} \otimes 1\right)+\left(b_{(1)} \otimes g\right) \otimes g \otimes\left(b_{(2)} \otimes g\right) \in \tilde{B} \otimes 1 \otimes \tilde{B}$ by composition with id $\otimes(\cdot \circ(\mathrm{S} \otimes \mathrm{id})) \otimes$ id. Thus $b=0$ and it follows that $A^{\prime} \subseteq A$. On the other hand clearly $A \subseteq A^{\prime}$ and thus $A=A^{\prime}$. This completes the proof.

The case of a general extension is obtained by considering two extensions of the spin generating group $\mathrm{Z}_{2}{ }^{\prime}$ with a surjection and then observing that the surjection survives the transition from the quantum group context to the $\mathbb{Z}_{2}$-graded group context.

Proposition 5.2. In the context of Theorem 5.1 consider two solutions of the quantum group extension problem with a surjection $A^{\prime} \rightarrow A \rightarrow \mathrm{Z}_{2}^{\prime}$. Then 
there is an induced surjection between the corresponding $\mathbb{Z}_{2}$-graded groups $B^{\prime} \rightarrow B \rightarrow H$. Thus, to the quantum group extension $A^{\prime} \rightarrow A$ corresponds the $\mathbb{Z}_{2}$-graded group extension $B^{\prime} \rightarrow B$.

In the case of ordinary quantum field theory the symmetry to be extended is the Poincaré group $B=\hat{\mathrm{P}}$ and it corresponds to the quantum group $A=\hat{\mathrm{P}}^{\prime}$ introduced in Section 4.1 (i.e., the Poincaré group with the cotriangular structure (14)). The statement of the Proposition 5.2 is now that any solution $A^{\prime} \rightarrow \hat{\mathrm{P}}^{\prime}$ of the quantum group extension problem is induced from a solution $B^{\prime} \rightarrow \hat{\mathrm{P}}^{\prime}$ of the $\mathbb{Z}_{2}$-graded group extension problem. But this is nothing but the supergroup extension problem described in the introduction. Thus, for ordinary QFT all the solutions of the quantum group extension problem are induced from solutions of the supergroup extension problem. The analysis of औ1 remains exhaustive in our generalized framework.

\section{Examples and Applications}

In this section we wish to demonstrate the usefulness of quantum geometric methods to supersymmetry. Notions of homogeneous space, quantum principal bundle, exterior derivative, all generalize from ordinary geometry to quantum geometry. In particular they apply to supergroups, superspaces, super-derivatives etc. Constructions in quantum geometry are just as easy for "super"-objects as they are for ordinary objects. Furthermore, they generalize far beyond the $\mathbb{Z}_{2}$-graded case (although we shall not consider this here), see in particular [5]. For example they apply to more general anyonic and braid statistics, see [6]. An application to fractional supersymmetry which fits into this framework is 11, 12.

\subsection{Elements of Quantum Geometry}

We start by introducing the basic notions and give elementary examples (from ordinary geometry). This section is mostly text book knowledge, see [5, 13]. For quantum principal bundles see [14], although our version here is more elementary. For statements from ordinary differential geometry, see e.g. [15].

Definition 6.1. Let $H$ be a Hopf algebra, $\beta: P \rightarrow P \otimes H$ a right $H$ comodule algebra. Set $B:=P^{H}=\{p \in P \mid \beta(p)=p \otimes 1\}$ and define $\chi$ : $P \otimes P \rightarrow P \otimes H$ by $\chi=(\cdot \otimes \mathrm{id}) \circ(\mathrm{id} \otimes \beta)$. If $\chi$ is surjective we call the triple $(P, B, H)$ a quantum principal bundle.

To see how this definition reduces to the usual one for ordinary principal bundles consider a group $G$ acting on a manifold $E$. This gives rise to a coaction $\beta: \mathcal{C}(E) \rightarrow \mathcal{C}(E) \otimes \mathcal{C}(G)$. The surjectivity of $\chi$ then means precisely 
that the map $G \times E \rightarrow E \times E$ defined by $(g, p) \mapsto(g p, p)$ is injective, i.e., that $G$ acts freely and thus defines a principal bundle The base space $M$ is the space of orbits of $G$ in $E$ and $\mathcal{C}(M)$ is precisely $\mathcal{C}(E)^{\mathcal{C}(G)}$.

Definition 6.2. Let $\pi: A \rightarrow H$ be a surjection of Hopf algebras. $A$ is a right $H$-comodule algebra by $\beta_{R}=(\mathrm{id} \otimes \pi) \circ \Delta$ and a left $H$-comodule algebra by $\beta_{L}=(\pi \otimes \mathrm{id}) \circ \Delta$. The space $A^{H}:=\left\{a \in A \mid \beta_{R}(a)=a \otimes 1\right\}$ forms a left $A$-comodule algebra via the coproduct of $A$ (and thus also a left $H$-comodule algebra via $\beta_{L}$ ). It is called a quantum homogeneous space.

Again we consider how this definition reduces to the one for ordinary homogeneous spaces. Let $G \hookrightarrow K$ be an injection of groups. This gives rise to a surjection of Hopf algebras $\pi: \mathcal{C}(K) \rightarrow \mathcal{C}(G)$. The functions on the homogeneous space $K / G$ by the induced action of $G$ on $K$ are precisely the functions on $K$ invariant under this group action, i.e., $\mathcal{C}(K / G) \cong \mathcal{C}(K)^{\mathcal{C}(G)}$. In particular, a homogeneous space gives rise to a principal bundle.

Remark 6.3. Given a Hopf algebra surjection $A \rightarrow H$ we obtain a quantum principal bundle $\left(A, A^{H}, H\right)$. Furthermore, this bundle is left $A$-equivariant, i.e., A carries a left coaction by $A$ itself which commutes with the right coaction of $H$ and thus descends to $A^{H}$.

This is in exact analogy to the situation in ordinary geometry.

Remark 6.4. Given a coquasitriangular Hopf algebra $H$ and a braided Hopf algebra in ${ }^{H} \mathcal{M}$ the bosonization gives rise to a Hopf algebra surjection $B \rtimes$ $H \rightarrow H$ (see Theorem 3.9). In particular, we obtain a quantum homogeneous space. In fact this precisely recovers $B$ itself (as an algebra) $B=(B \rtimes H)^{H}$.

This generalizes the situation of a semidirect product of Lie groups giving rise to a homogeneous space.

Remark 6.5. Note that for a homogeneous space $K / G$ the principal bundle $(K, K / G, G)$ can be identified with (a reduction of) the frame bundle on $K / G$. Furthermore, if $K / G$ is Riemannian such that $K$ consists of isometries, the bundle $(K, K / G, G)$ can be identified with (a reduction of) the bundle of orthonormal frames on $K / G$. Now, if $K / G$ is orientable the bundle decomposes into two connected components (corresponding to the two possible orientations). We take the one corresponding to the orientation preserving subgroup of $K$ and denote it $\left(K^{\prime}, K / G, G^{\prime}\right)$. K/G admits a spin structure iff $K^{\prime}$ admits a double cover $\hat{K}$. The spin bundle is then $(\hat{K}, K / G, \hat{G})$ where $\hat{G}$ is the corresponding double cover of $G^{\prime}$.

\footnotetext{
${ }^{6}$ In fact, it is also necessary that $G$ acts properly. This is a somewhat technical condition, strongly related to the class of functions we consider. It is satisfied for "good" cases such as when $G$ is compact. We thus consider this condition as "not being visible" in our algebraic setting.
} 
Example 6.6. Consider $S^{2}$ as the homogeneous space $S O(3) / S O(2)=S U(2) / U(1)$. The injection $U(1) \hookrightarrow S U(2)$ becomes a surjection $\mathrm{SU}(2) \rightarrow \mathrm{U}(1)$ from the quantum group point of view. $\mathrm{U}(1)$ has a (Fourier, i.e., Peter-Weyl) basis $\left\{g^{m}\right\}$ with $m \in \mathbb{Z}$, coproduct $\Delta g^{m}=g^{m} \otimes g^{m}$, counit $\epsilon(g)=1$, and antipode $\mathrm{S} g^{m}=g^{-m}$. The Hopf algebra surjection is given by

$$
t_{m n}^{l} \mapsto g^{2 m} \delta_{m n}
$$

in the basis of Example 3.1 for $\mathrm{SU}(2)$.

The surjection (18) induces the right coaction $t_{m n}^{l} \mapsto t_{m n}^{l} \otimes g^{2 n}$ of $\mathrm{U}(1)$ on $\mathrm{SU}(2)$ leading to the algebra $\mathrm{S}^{2}$ as its right invariant subspace. A basis of it is given by $\left\{t_{n 0}^{l}\right\}$ with $l \in N_{0}$. These are precisely the spherical harmonics on $S^{2}$.

Note that according to Remark 6.5 we can view $\left(\mathrm{SU}(2), \mathrm{S}^{2}, \mathrm{U}(1)\right)$ as the spin-bundle on $\mathrm{S}^{2}$.

We now turn to the concept of quantum differentials that generalizes the concepts of differential 1 -forms and vector fields to the quantum geometric realm.

Definition 6.7 ( $\llbracket \mathbf{1 6} \rrbracket)$. Let $A$ be a Hopf algebra. Let $\Omega$ be a bicovariant bimodule over $A$. That is, a left and right $A$-module and a left and right $A$-comodule, such that actions and coactions commute in the obvious ways. Assume there is a bicomodule map $\mathrm{d}: A \rightarrow \Omega$. That is, $\mathrm{d}$ is a left and right A-comodule map. If the Leibniz rule

$$
\mathrm{d}(a b)=\mathrm{d}(a) b+a \mathrm{~d}(b), \quad \forall a, b \in A
$$

holds and the map $A \otimes A \rightarrow \Omega$ given by $a \otimes b \mapsto a \mathrm{~d} b$ is surjective, then we call $\Omega$ a (bicovariant first-order) differential calculus.

Proposition 6.8 ([16], see also [17]). Let $A$ and $H$ be Hopf algebras that are non-degenerately dually paired. Then, differential calculi on A correspond to subspaces of $L \subseteq H^{\prime}:=\operatorname{ker} \epsilon \subset H$ with the following properties:

(a) $L$ is invariant under the left coaction of $H$ defined by $\eta \mapsto \eta_{(1)} \otimes$ $\eta_{(2)}-\eta \otimes 1$. That is, the coaction $H^{\prime} \rightarrow H \otimes H^{\prime}$ defined in this way descends to $L \rightarrow H \otimes L$.

(b) $L$ is invariant under the left action of $H$ given by $h \triangleright \eta=h_{(1)} \eta \mathrm{S} h_{(2)}$. That is, the action $H \otimes H^{\prime} \rightarrow H^{\prime}$ defined in this way restricts to $H \otimes L \rightarrow L$.

The space $L$ can be thought of as the space of right-invariant vector fields that act on $A$ as "derivatives" from the right via

$$
L \otimes A \rightarrow A: \eta \otimes a \mapsto \partial_{\eta}(a):=\left\langle\eta, a_{(1)}\right\rangle a_{(2)}
$$

for $\eta \in L$ and $a \in A$. Dually, $\Gamma:=L^{*}$ is the space of right-invariant 1-forms. $\Gamma$ carries a left action of $A$ determined by the coaction (a) via

$$
\langle\eta, a \triangleright \omega\rangle=\left\langle\eta_{(1)}, a\right\rangle\left\langle\eta_{(2)}, \omega\right\rangle-\langle\eta, a\rangle\langle 1, \omega\rangle
$$


for $a \in A, \eta \in L, \omega \in \Gamma$. $\Gamma$ carries a left coaction $\omega \mapsto \omega_{[1]} \otimes \omega_{[2]}$ of $A$ determined by the action (b) via

$$
\left\langle h, \omega_{[1]}\right\rangle\left\langle\eta, \omega_{[\underline{2]}}\right\rangle=\left\langle h_{(1)} \eta \mathrm{S} h_{(2)}, \omega\right\rangle
$$

for $h \in H, \eta \in L, \omega \in \Gamma$. The corresponding differential calculus $\Omega$ on $A$ is isomorphic to $\Gamma \otimes A$ as a vector space. Its right $A$-module and comodule structure are given by multiplication and comultiplication on A. Its left Amodule and comodule structure are the tensor product ones. The map $d$ is recovered from the derivative as $\mathrm{d}(a)=\sum_{i} \omega_{i} \partial_{\eta_{i}}(a)$ with $\eta_{i}$ a basis of $L$ and $\omega_{i}$ the dual basis of $\Gamma$.

The left coaction $\eta \mapsto \eta_{[1]} \otimes \eta_{[2]}$ of $A$ on $L$ that makes the derivative map $L \otimes A \rightarrow A$ covariant is determined through the action of condition (b) by

$$
\left\langle\mathrm{S} h, \eta_{[1]}\right\rangle \eta_{[2]}=h_{(1)} \eta \mathrm{S} h_{(2)} .
$$

for $h \in H, \eta \in L$.

Remark 6.9. Let $G$ be a Lie group and $\mathfrak{g}$ its Lie algebra. We set $A=\mathcal{C}(G)$ and $H=\mathcal{U}(\mathfrak{g})$ above and recover the usual differentials with $L=\mathfrak{g}$. Note that the coaction of condition (a) becomes trivial while the action of condition (b) is by the Lie bracket.

Example 6.10. Consider the dually paired quantum groups $\mathrm{SU}(2)$ and $\mathcal{U}\left(\mathfrak{s u}_{2}\right)$ described in Example 3.3. The ordinary tangent space is $\mathfrak{s u}_{2}$ with basis $E, F, H$ leading to the derivatives

$$
\begin{gathered}
\partial_{H}\left(t_{m n}^{l}\right)=2 m t_{m n}^{l}, \quad \partial_{E}\left(t_{m n}^{l}\right)=\sqrt{(l-m+1)(l+m)} t_{m-1, n}^{l} \\
\partial_{F}\left(t_{m n}^{l}\right)=\sqrt{(l+m+1)(l-m)} t_{m+1, n}^{l} .
\end{gathered}
$$

Note that this result extends to the dually paired quantum groups $\operatorname{Spin}(3,1)$ and $\mathcal{U}\left(\mathfrak{s o}_{3,1}\right)$ precisely as in the transition from Example 3.9 to Example 3.4.

Example 6.11. Consider the dually paired Poincaré group Poinc and its Lie algebra poinc of Examples 3.19 and 3.13. The derivatives given by poinc on Poinc come out as in Example 6.10 supplemented by

$$
\begin{gathered}
\partial_{X}\left(x^{\mu}\right)=\frac{1}{2} \sum_{\nu} \operatorname{tr}\left(\sigma^{\mu} \sigma(X) \sigma^{\nu}\right), \quad \partial_{\bar{X}}\left(x^{\mu}\right)=\overline{\partial_{X}\left(x^{\mu}\right)}, \quad \forall X \in\{H, E, F\} \\
\partial_{P^{\mu}}\left(t_{m n}^{l}\right)=\partial_{P^{\mu}}\left(\overline{t_{m n}^{l}}\right)=0, \quad \partial_{P^{\mu}}\left(x^{\nu}\right)=\delta^{\mu \nu} .
\end{gathered}
$$




\subsection{Semidirect Superextensions}

In this section we consider semidirect superextensions which are simple examples of superextensions. These serve at the same time as a preparation for the more involved superextensions considered later.

For our present mathematical purposes we give the following definition of "superextension" as a minor modification of Definition 3.8.

Definition 6.12. Let $H$ be a coquasitriangular Hopf algebra. Then $A$ is called a finite non-trivial extension or superextension of $H$ if (a) $A \rightarrow H$ is a surjection of coquasitriangular Hopf algebras, (b) there is no Hopf algebra $K$ such that $A \cong H \otimes K$ as a Hopf algebra and, (c) $A^{H}$ is finite dimensional.

Remark 6.13. In the quantum principal bundle picture $\left(A, A^{H}, H\right)$ condition (b) corresponds to the bundle being non-trivial. Condition (c) says that the algebra of functions on the base space is finite dimensional or the base space itself "zero-dimensional". Physically speaking it means that the number of superfield components is finite.

We shall be interested in the case where $H$ is a cotriangular Hopf algebra of the type of Lemma 4.1 as this is the physically interesting situation (see Section (1). Dually, we shall as well consider dual superextensions (defined in the obvious way) for triangular Hopf algebras of the type of Lemma 4.3.

An important (well known) supergroup is the analog of the translation group on $\mathbb{R}^{n}$ defined as follows.

Lemma 6.14. Let $\Theta_{n}$ be the unital algebra generated by $\left\{\theta_{1}, \ldots, \theta_{n}\right\}$ with relations $\theta_{i} \theta_{j}=-\theta_{j} \theta_{i}$. It is $\mathbb{Z}_{2}$-graded by $|1|=0,\left|\theta_{i}\right|=1$. It extends to a $\mathbb{Z}_{2}$-graded commutative Hopf algebra by the coalgebra structure $\Delta \theta_{i}=$ $1 \otimes \theta_{i}+\theta_{i} \otimes 1$. Counit and antipode are given by $\epsilon\left(\theta_{i}\right)=0$ and $\mathrm{S} \theta_{i}=-\theta_{i}$.

Furthermore, $\Theta_{n}$ is self-dual via the pairing generated by $\left\langle\theta_{i}, \theta_{j}\right\rangle=\delta_{i j}$.

We are now ready to consider semidirect superextensions.

Proposition 6.15. In the context of Lemma 4.1 let $V$ be a finite dimensional comodule of $H$ such that for the coaction $\beta: V \rightarrow H \otimes V$ the condition $\beta(V) \subseteq H_{1} \otimes V$ holds. Then, $\Theta_{V}:=\Theta_{n}$ with $\left\{\theta_{1}, \ldots, \theta_{n}\right\}$ a basis of $V$ is a braided $H$-comodule Hopf algebra.

Furthermore, the semidirect product (bosonization) $\Theta_{V} \rtimes H$ is a superextension of $H$. It is generated as an algebra by $H$ and $\theta_{i}$ with relations $\theta_{i} \theta_{j}=-\theta_{j} \theta_{i}$ and $h \theta_{i}=(-1)^{|h|} \theta_{i} h$. The coalgebra structure on the $H$ is the given one and for $\theta_{i}$ we have

$$
\Delta \theta_{i}=\theta_{i} \otimes 1+\beta\left(\theta_{i}\right) .
$$

\footnotetext{
${ }^{7}$ Note that we take the pairing here in the usual sense for Hopf algebras and not the usual sense for braided Hopf algebras. That is, we require $\langle\phi, v w\rangle=\left\langle\phi_{(1)}, v\right\rangle\left\langle\phi_{(2)}, w\right\rangle$ etc., and not with $\phi_{(1)}, \phi_{(2)}$ interchanged.
} 
Corollary 6.16. Let $H$ be a commutative Hopf algebra generated by the $n$ dimensional matrix coalgebra $T$ and equipped with the cotriangular structure

$$
\mathcal{R}\left(t_{i j} \otimes t_{k l}\right)=-\epsilon\left(t_{i j}\right) \epsilon\left(t_{k l}\right)=-\delta_{i j} \delta_{k l} .
$$

Then, $\Theta_{n}$ is a (braided) H-comodule Hopf algebra.

Furthermore, the semidirect product (bosonization) $\Theta_{n} \rtimes H$ is a superextension of $H$. It is generated as an algebra by $t_{i j}$ and $\theta_{i}$ with relations $\theta_{i} \theta_{j}=-\theta_{j} \theta_{i}$ and $\theta_{i} t_{j k}=-t_{j k} \theta_{i}$. The coalgebra structure on the $t_{i j}$ is the matrix coalgebra structure while for $\theta_{i}$ we obtain

$$
\Delta \theta_{i}=\theta_{i} \otimes 1+\sum_{j} t_{i j} \otimes \theta_{j}
$$

Remark 6.17. Note that while the above seems to be adapted to real matrix groups it works equally well for complex matrix groups. In that case, double the range of the indices and define $t_{i+n, j+n}:=\overline{t_{i, j}}$ and set $t_{i, j+n}=0=t_{i+n, j}$.

We can equally consider the dual setting with the "enveloping algebra" counterpart of $H$, although we need to adjoin an extra generator in this case as described in Section 4.2 .

Proposition 6.18. In the context of Lemma 4.5 given an $n$-dimensional $A$-module $V$ with basis $\left\{Q_{1}, \ldots, Q_{n}\right\}$ such that $\xi \triangleright Q_{i}=-Q_{i}$, it extends to the (braided) A-module Hopf algebra $\Theta_{V}:=\Theta_{n}$.

Furthermore, the semidirect product (bosonization) $\Theta_{V} \rtimes A$ is a dual superextension of $A$. It is generated as an algebra by $A$ and $\Theta_{V}$ with crossrelations $a Q_{i}=\left(a_{(1)} \triangleright Q_{i}\right) a_{(2)} \forall a \in A$. For primitive elements of $A$ the latter take the commutator form $\left[a, Q_{i}\right]=a \triangleright Q_{i}$. The coalgebra structure on $A$ is the given one and for $Q_{i}$ we obtain $\Delta Q_{i}=Q_{i} \otimes 1+\xi \otimes Q_{i}$.

Example 6.19. Consider the superextension $\Theta_{2} \rtimes \mathcal{U}^{\prime}\left(\mathfrak{s u}_{2}\right)$ where $\Theta_{2}$ is in the fundamental representation. Explicitly, we denote a basis of $\Theta_{2}$ by $\left\{Q_{ \pm}\right\}$ and define the action with $\sigma(X)$ given as in Example 3.4 as

$$
\begin{gathered}
X \triangleright Q_{i}=\sum_{j} \sigma(X)_{j i} Q_{j} \quad \forall X \in\{H, E, F\}, \quad \text { or explicitly, } \\
E \triangleright Q_{+}=0, \quad E \triangleright Q_{-}=Q_{+}, \quad F \triangleright Q_{+}=Q_{-}, \quad F \triangleright Q_{-}=0, \\
H \triangleright Q_{ \pm}= \pm Q_{ \pm}, \quad \xi \triangleright Q_{ \pm}=-Q_{ \pm} .
\end{gathered}
$$

The cross-relations are immediate from that:

$$
\begin{gathered}
{\left[E, Q_{+}\right]=0, \quad\left[E, Q_{-}\right]=Q_{+}, \quad\left[F, Q_{+}\right]=Q_{-}, \quad\left[F, Q_{-}\right]=0} \\
{\left[H, Q_{ \pm}\right]= \pm Q_{ \pm}, \quad \xi Q_{ \pm}=-Q_{ \pm} \xi .}
\end{gathered}
$$


Example 6.20. The pairing of $\Theta_{2} \rtimes \mathrm{SU}^{\prime}(2)$ and $\Theta_{2} \rtimes \mathcal{U}^{\prime}\left(\mathfrak{s l}_{2}\right)$ (using Lemma 3.11) leads to the natural quantum tangent space with basis $E, F, H, Q_{+}, Q_{-}$. The corresponding derivatives are the ones of Example 6.10 extended by

$$
\begin{array}{rll}
\partial_{E}\left(\theta_{+}\right)=\theta_{-}, \quad \partial_{E}\left(\theta_{-}\right)=0, & \partial_{F}\left(\theta_{+}\right)=0, \quad \partial_{F}\left(\theta_{-}\right)=\theta_{+} \\
\partial_{H}\left(\theta_{ \pm}\right)= \pm \theta_{ \pm}, & \partial_{Q_{ \pm}}\left(t_{m n}^{l}\right)=0, \\
\partial_{Q_{+}}\left(\theta_{+}\right)=1, \quad \partial_{Q_{+}}\left(\theta_{-}\right)=0, & \partial_{Q_{-}}\left(\theta_{+}\right)=0, \quad \partial_{Q_{-}}\left(\theta_{-}\right)=1
\end{array}
$$

For homogeneous spaces we can easily obtain their extensions to superspaces corresponding to superextensions of the group. This is simply the corresponding quantum homogeneous space.

Example 6.21. Analogous to the ordinary 2-sphere as a homogeneous space of $S U(2)$ (Example 6.6) we can build a supersymmetric version as the quantum homogeneous space of $\Theta_{2} \rtimes \mathrm{SU}^{\prime}(2)$ via the surjection $\pi: \Theta_{2} \rtimes \mathrm{SU}^{\prime}(2) \rightarrow$ $\mathrm{U}(1)$. We call this the semidirect super-sphere. $\pi$ is simply given by the extension map $\Theta_{2} \rtimes \mathrm{SU}^{\prime}(2) \rightarrow \mathrm{SU}^{\prime}(2)$ composed with the usual surjection $\mathrm{SU}^{\prime}(2) \rightarrow \mathrm{U}^{\prime}(1)$ given by (8). Note that this is a cotriangular Hopf algebra map upon choosing $\mathcal{R}\left(g^{m} \otimes g^{n}\right)=(-1)^{m n} \delta_{m n}$ on $\mathrm{U}^{\prime}(1)$ (hence the prime in the notation) corresponding to $U(1)$ covering itself twice. Now the semidirect super-sphere is simply the subalgebra $\mathrm{S}_{2}^{2}$ of $\Theta_{2} \rtimes \mathrm{SU}^{\prime}(2)$ which is (right) invariant under the coaction induced by $\pi$. That is, it is the subalgebra with basis $\left\{t_{i, 0}^{l}, t_{i,-}^{l} \theta_{+}, t_{i,+}^{l} \theta_{-}, t_{i, 0}^{l} \theta_{+} \theta_{-}\right\}$.

Note that $\mathrm{S}_{2}^{2}$ is a left $\Theta_{2} \rtimes \mathrm{SU}^{\prime}(2)$-comodule algebra (via the coproduct) by construction. It gives rise to a quantum principal bundle $\left(\Theta_{2} \rtimes\right.$ $\mathrm{SU}^{\prime}(2), \mathrm{S}_{2}^{2}, \mathrm{U}^{\prime}(1)$ ) (see Remark 6.3). Upon "reducing the base space" to $\mathrm{S}^{2}$ it becomes the spin-bundle $\left(\mathrm{SU}^{\prime}(2), \mathrm{S}^{2}, \mathrm{U}^{\prime}(1)\right)$ of $\mathrm{S}^{2}$. Thus, we can view it as the spin-bundle of $\mathrm{S}_{2}^{2}$.

\subsection{Matrix Supergroups}

We now consider more complicated superextensions which are super-analogues of matrix groups. These are well known in the theory of supergroups, see e.g. [18]. (However, our setting is closer in spirit to [19].) Much of their treatment here is along the lines of [8] and [5] (where even more general braidings are considered). However, the "physical" quantum group versions of supergroups (motivated from Section 4) seem not to have been considered previously. In particular, the quantum group version $\mathrm{M}^{\prime}(m \mid n)$ as well as the treatment of the $O S p$-supergroups in this context appear to be new.

Let $m, n$ be natural numbers. Consider a $\mathbb{Z}_{2}$-graded vector space $V$ with basis $\left\{v_{i}\right\}$ for $i \in\{1, \ldots, m+n\}$ such that $\left|v_{i}\right|:=|i|$ with

$$
|i|:=\left\{\begin{array}{ll}
1 & \text { if } i \in\{1, \ldots, m\} \\
0 & \text { if } i \in\{m+1, \ldots, m+n\}
\end{array} .\right.
$$


Assume further that a graded commutative Hopf algebra (i.e., a supergroup) $H$ coacts (graded) from the left on $V$. Explicitly, $v_{i} \mapsto \sum_{j} u_{i j} \otimes v_{j}$. As the coaction is graded the grading on the elements $\left\{u_{i j}\right\}$ must be given by $\left|u_{i j}\right|=|i|+|j|$.

Conversely, in order to construct such a graded commutative Hopf algebra, we start (exactly as we would do in the theory of matrix groups) with the universal graded commutative bialgebra that coacts on $V$.

Definition 6.22. Consider the matrix coalgebra generated by $\left\{u_{i j}\right\}$ with $i, j \in\{1, \ldots, m+n\}$. It becomes a $\mathbb{Z}_{2}$-graded coalgebra (i.e., coproduct and counit respect the grading) by defining $\left|u_{i j}\right|:=|i|+|j|$ (mod 2 understood). Next, consider its tensor algebra and extend the coproduct to it as a graded algebra map. We obtain a graded bialgebra. Finally, we quotient by the graded commutativity relation

$$
u_{i j} u_{k l}=(-1)^{(|i|+|j|)(|k|+|l|)} u_{k l} u_{i j} .
$$

As this is compatible with the coproduct we obtain a graded commutative bialgebra $\mathrm{M}(m \mid n)$. We call it the matrix super-bialgebra of rank $(m \mid n)$.

Note that we can quotient by the relations $u_{i j}=0$ for $|i| \neq|j|$ to obtain a tensor product of purely even commutative matrix bialgebras

$$
\mathrm{M}(m \mid n) \rightarrow \mathrm{M}(m) \otimes \mathrm{M}(n) .
$$

In the above context of a graded commutative Hopf algebra coacting on a graded vector space $V$ we consider the dual space $V^{*}$ with a pairing $V \otimes V^{*} \rightarrow \mathbb{C}$. It naturally becomes a graded $H$-comodule by the coaction $v_{i}^{*} \mapsto \sum_{j}(-1)^{|j| \cdot(|j|+|i|)} \mathrm{S} u_{j i} \otimes v_{j}^{*}$ which leaves the pairing invariant (with $\left\{v_{i}^{*}\right\}$ the dual basis to $\left.\left\{v_{i}\right\}\right)$.

Assume now that $V$ and $V^{*}$ are isomorphic as graded $H$-comodules via a map $\eta: V^{*} \rightarrow V$ with $v_{i}^{*} \mapsto \sum_{j} \eta_{i j} v_{j}$. As $\eta$ is bijective the inverse matrix $\eta_{i j}^{-1}$ exists and $\eta_{i j}=\eta_{i j}^{-1}=0$ for $|i| \neq|j|$ as it is graded. The fact that $\eta$ is a comodule map implies that the elements $\mathrm{S} u_{i j}$ of $H$ can be written in terms of the $u_{i j}$ as

$$
\mathrm{S} u_{i j}=\sum_{k, l}(-1)^{|l| \cdot(|l|+|j|)} \eta_{j k} u_{k l} \eta_{l i}^{-1}
$$

This in turn implies the relations

$$
\begin{aligned}
\eta_{k l} \mathbf{1} & =\sum_{i, j}(-1)^{|l| \cdot(|l|+|i|)} \eta_{i j} u_{j l} u_{i k} \\
\eta_{k l}^{-1} \mathbf{1} & =\sum_{i, j}(-1)^{|i| \cdot(|i|+|k|)} u_{l j} u_{k i} \eta_{i j}^{-1}
\end{aligned}
$$

by the defining property of the antipode. 
Conversely, we can construct a graded Hopf algebra by demanding it to be the universal graded Hopf algebra with coaction on $V$ such that $\eta$ is a graded comodule map. This is analogous to constructing a matrix group that leaves a non-degenerate bilinear form on its defining representation (which can be seen as an isomorphism to the dual representation) invariant.

Proposition 6.23. Let $\eta_{i j}$ be an invertible matrix such that $\eta_{i j}=0$ if $|i| \neq|j|$. Consider the graded commutative bialgebra $\mathrm{M}(m \mid n)$ and impose the relations (19) and (13). They are compatible with the coalgebra structure so that we obtain again a graded commutative bialgebra $\operatorname{lnv}_{\eta}(m \mid n)$. Furthermore, $\operatorname{lnv}_{\eta}(m \mid n)$ is a graded Hopf algebra with antipode given by (11).

Example 6.24. Let $r, s$ be natural numbers. Define an invertible matrix $\eta_{i j}$ of rank $2 r+s$ by

$$
\begin{gathered}
\eta_{2 i-1,2 i}=1, \quad \eta_{2 i, 2 i-1}=-1, \quad \text { for } i \in\{1, \cdots, r\}, \\
\eta_{j, j}=1, \quad \text { for } j \in\{m+1, \ldots, m+n\},
\end{gathered}
$$

and all other entries zero. The graded commutative Hopf algebra $\operatorname{Inv}_{\eta}(2 r \mid s)$ is called the ortho-symplectic supergroup $\operatorname{OSp}(2 r \mid s)$. Its even commutative quotient is

$$
\mathrm{OSp}(2 r \mid s) \rightarrow \mathrm{Sp}(2 r) \otimes \mathrm{O}(s)
$$

Example 6.25. $\mathrm{OSp}(2 \mid 1)$ is the matrix super-bialgebra $\mathrm{M}(2 \mid 1)$ with additional relations

$$
\begin{gathered}
u_{13} u_{23}-u_{31} u_{32}=0, \\
2 u_{32} u_{31}+u_{33} u_{33}=1, \\
u_{11} u_{22}-u_{12} u_{21}+u_{13} u_{23}=1, \\
-u_{11} u_{32}+u_{12} u_{31}+u_{13} u_{33}=0, \\
-u_{21} u_{32}+u_{22} u_{31}+u_{23} u_{33}=0, \\
u_{22} u_{13}-u_{12} u_{23}-u_{32} u_{33}=0, \\
-u_{21} u_{13}+u_{11} u_{23}+u_{31} u_{33}=0 .
\end{gathered}
$$

It has an antipode given by

$$
\mathrm{S}\left(\begin{array}{lll}
u_{11} & u_{12} & u_{13} \\
u_{21} & u_{22} & u_{23} \\
u_{31} & u_{32} & u_{33}
\end{array}\right)=\left(\begin{array}{ccc}
u_{22} & -u_{12} & -u_{32} \\
-u_{21} & u_{11} & u_{31} \\
u_{23} & -u_{13} & u_{33}
\end{array}\right) .
$$

Let us now consider the corresponding quantum groups (i.e., cotriangular Hopf algebras). According to Theorem 3.9 and Proposition 3.10 the cotriangular bialgebra with the same representation category as $\mathrm{M}(m \mid n)$ is 
the bosonization $\mathrm{M}(m \mid n) \rtimes \mathbf{Z}_{2}{ }^{\prime}$. It is generated by $\mathrm{M}(m \mid n)$ and $\mathbf{Z}_{2}{ }^{\prime}$ as an algebra with cross relations $g u_{i j}=(-1)^{|i|+|j|} u_{i j} g$ and has coproduct

$$
\Delta u_{i j}=\sum_{k} u_{i k} g^{|k|+|j|} \otimes u_{k j}, \quad \Delta g=g \otimes g .
$$

If we want to consider $\mathrm{M}(m \mid n) \rtimes \mathrm{Z}_{2}^{\prime}$ as a symmetry of a quantum field theory with Bose-Fermi statistics we need to perform the spin-statistics reduction (Section 4, Definition 4.5) in order to eliminate representations with the wrong spin-statistics relation. In fact, this is already suggested by our construction. The fact that the vector space $V$ has $m$ odd and $n$ even basis vectors and not the other way round is "forgotten" by the super matrixbialgebra $\mathrm{M}(m \mid n)$ as $\mathrm{M}(m \mid n) \cong \mathrm{M}(n \mid m)$. However, writing the coaction of the bosonization $\mathrm{M}(m \mid n) \rtimes \mathrm{Z}_{2}^{\prime}$ on $V$ we find

$$
v_{i} \mapsto \sum_{j} u_{i j} g^{|j|} \otimes v_{j}
$$

This suggest that we should in the commutative quotient (10) interpret $\mathrm{M}(m)$ as generating the spin. Then (14) precisely reflects the fact that $V$ has the right spin-statistics relation. Conversely, the spin-statistics reduction of $\mathrm{M}(m \mid n) \rtimes \mathrm{Z}_{2}^{\prime}$ is given by its sub-bialgebra generated by $u_{i j} g^{|j|}$.

Apart from the physical motivation, we can also motivate this reduction purely mathematically by demanding that we want to consider the universal object coacting on $V$.

Proposition 6.26. The spin-statistics reduction $\mathrm{M}^{\prime}(m \mid n)$ of $\mathrm{M}(m \mid n) \rtimes \mathrm{Z}_{2}{ }^{\prime}$ is given by its sub-bialgebra generated by the elements $t_{i j}:=u_{i j} g^{|j|}$. Explicitly, it is generated by the matrix coalgebra $\left\{t_{i j}\right\}$ with relations given by the cotriangular structure

$$
\mathcal{R}\left(t_{i j} \otimes t_{k l}\right)=(-1)^{|i| \cdot|k|} \delta_{i j} \delta_{k l}
$$

Explicitly,

$$
t_{i j} t_{k l}=(-1)^{|i| \cdot|k|+|j| \cdot|l|} t_{k l} t_{i j}
$$

It has a commutative quotient

$$
\mathrm{M}^{\prime}(m \mid n) \rightarrow \mathrm{M}^{\prime}(m) \otimes \mathrm{M}(n)
$$

by $t_{i j} \mapsto 0$ for $|i| \neq|j|$. This is a map of cotriangular bialgebras where $\mathrm{M}^{\prime}(m)$ has cotriangular structure (9) and $\mathrm{M}(n)$ has trivial cotriangular structure. Furthermore, it is a (bialgebra) superextension in the sense of Definition 6.19. 
Note that choosing the "wrong" spin-statistics relation also yields a subbialgebra of $\mathrm{M}(m \mid n) \rtimes \mathrm{Z}_{2}^{\prime}$ which is generated by $\left\{u_{i j} g^{|j|+1}\right\}$. It is isomorphic to $\mathrm{M}^{\prime}(n \mid m)$.

Let us now consider the spin-statistics reduction of the bosonization of a graded Hopf algebra $\operatorname{lnv}_{\eta}(m \mid n) \rtimes \mathbf{Z}_{2}^{\prime}$ that leaves an isomorphism $\eta: V^{*} \rightarrow V$ invariant. In fact, instead of constructing first $\operatorname{lnv}_{\eta}(m \mid n)$ and then its spinstatistics reduction we can proceed directly. Thus, the dual space $V^{*}$ carries naturally a coaction $v_{i}^{*} \mapsto \sum_{j} \mathrm{~S} t_{j i} \otimes v_{j}^{*}$ in terms of the antipode. Now, an isomorphism $\eta: V^{*} \rightarrow V$ as above implies for the antipode

$$
\mathrm{S} t_{i j}=\sum_{k, l} \eta_{j k} t_{k l} \eta_{l i}^{-1}
$$

and thus the relations

$$
\begin{aligned}
\eta_{k l} \mathbf{1} & =\sum_{i, j} \eta_{i j} t_{j l} t_{i k}, \\
\eta_{k l}^{-1} \mathbf{1} & =\sum_{i, j} t_{l j} t_{k i} \eta_{i j}^{-1} .
\end{aligned}
$$

Alternatively, these are obtained from (11 13) by using $u_{i j}=t_{i j} g^{|j|}$ and the commutation relation with $g$ in $\mathrm{M}(m \mid n) \rtimes \mathrm{Z}_{2}^{\prime}$.

Proposition 6.27. Let $\eta_{i j}$ be an invertible matrix such that $\eta_{i j}=0$ if $|i| \neq|j|$. Consider the cotriangular bialgebra $\mathrm{M}^{\prime}(m \mid n)$ and impose the relations (10) and (17). They are compatible with the coalgebra structure and cotriangular structure so that we obtain again a cotriangular bialgebra $\operatorname{Inv}_{\eta}^{\prime}(m \mid n)$. Furthermore, $\operatorname{Inv}_{\eta}^{\prime}(m \mid n)$ is a cotriangular Hopf algebra with antipode given by (11).

We denote the cotriangular Hopf algebra version of the ortho-symplectic supergroup by $\mathrm{OSp}^{\prime}(2 r \mid s)$. Its commutative quotient gives rise to the superextension

$$
\mathrm{OSp}^{\prime}(2 r \mid s) \rightarrow \mathrm{Sp}^{\prime}(2 r) \otimes \mathrm{O}(s)
$$

Thus, physically, the spin is attached to the symplectic group $\mathrm{Sp}^{\prime}(2 r)$. Note however, that we can construct a second version $\mathrm{OSp}^{\prime \prime}(2 r \mid s)$ based on $\mathrm{M}^{\prime}(s \mid 2 r)$ which has a commutative quotient

$$
\mathrm{OSp}^{\prime \prime}(2 r \mid s) \rightarrow \mathrm{Sp}(2 r) \otimes \mathrm{O}^{\prime}(s) .
$$

In this case the spin is attached to the orthogonal group $\mathrm{O}^{\prime}(s)$.

Example 6.28. $\operatorname{OSp}^{\prime}(2 \mid 1)$ is the cotriangular matrix bialgebra $\mathrm{M}^{\prime}(2 \mid 1)$ with additional relations

$$
t_{13} t_{23}+t_{31} t_{32}=0
$$




$$
\begin{gathered}
2 t_{31} t_{32}+t_{33} t_{33}=1, \\
t_{11} t_{22}-t_{12} t_{21}+t_{13} t_{23}=1, \\
t_{11} t_{32}-t_{12} t_{31}+t_{13} t_{33}=0, \\
t_{21} t_{32}-t_{22} t_{31}+t_{23} t_{33}=0, \\
t_{22} t_{13}-t_{12} t_{23}+t_{32} t_{33}=0, \\
t_{21} t_{13}-t_{11} t_{23}+t_{31} t_{33}=0 .
\end{gathered}
$$

Its antipode is given by

$$
\mathrm{S}\left(\begin{array}{ccc}
t_{11} & t_{12} & t_{13} \\
t_{21} & t_{22} & t_{23} \\
t_{31} & t_{32} & t_{33}
\end{array}\right)=\left(\begin{array}{ccc}
t_{22} & -t_{12} & t_{32} \\
-t_{21} & t_{11} & -t_{31} \\
t_{23} & -t_{13} & t_{33}
\end{array}\right) .
$$

Example 6.29. Consider the quantum homogeneous space given by $\pi$ : $\operatorname{OSp}^{\prime}(2 \mid 1) \rightarrow \mathrm{U}^{\prime}(1)$. This is another version of the super-sphere (see 201) which we call the OSp(2|1) super-sphere. Here, $\pi$ is defined as the composition of the extension map (18) with id $\otimes \epsilon$ and subsequently with (8). A set of generators of this subalgebra $\mathrm{S}^{2 \mid 1}$ of $\mathrm{OSp}^{\prime}(2 \mid 1)$ is given by $\left\{t_{i 3}, t_{i 1} t_{j 2}\right\}$. (The relations are as in Example 6.28.)

Analogous to Example 6.21 we can view $\left(\mathrm{OSp}^{\prime}(2 \mid 1), \mathrm{S}^{2 \mid 1}, \mathrm{U}^{\prime}(1)\right)$ as the spin-bundle over $\mathrm{S}^{2 \mid 1}$.

For example super-Anti-de-Sitter space can be constructed precisely in this way. As Anti-de-Sitter space is the homogeneous space $S O(3,2) / S O(3,1)$ we pass to the spin groups $\operatorname{Spin}(3,2) / \operatorname{Spin}(3,1)$ where $\operatorname{Spin}(3,2)=S p(4)$. Then we consider the "physical" quantum groups $\operatorname{Sp}^{\prime}(4) \rightarrow \operatorname{Spin}^{\prime}(3,1)$ and the superextension $\mathrm{OSp}^{\prime}(4 \mid 1) \rightarrow \mathrm{Sp}^{\prime}(4)$. The corresponding superextension of Anti-de-Sitter space is thus the quantum homogeneous space OSp $\mathbf{O S}^{\prime}(4 \mid 1)^{\mathrm{Sp}^{\prime}(4)}$.

\subsection{The Super-Poincaré Group}

In this section we consider the standard super-Poincaré group which is yet another type of superextension. Its presentation here in the quantum geometric framework has some novel aspects (in particular, the consideration of the "physical" quantum group version).

Recall the context of Example 3.12. We start with the proper quantum mechanical version of the Lorentz group $\operatorname{Spin}(3,1)$ which is equipped with the cotriangular structure $\mathcal{R}\left(t_{i j} \otimes t_{k l}\right)=-\delta_{i j} \delta_{k l}$ (same if one or both $t$ carry a bar) of Bose-Fermi statistics (see Section 4.1) and which we denote by $\operatorname{Spin}^{\prime}(3,1)$. Correspondingly, the quantum mechanical Poincare group is the quantum group Poinc ${ }^{\prime}=$ Mink $\rtimes \operatorname{Spin}^{\prime}(3,1)$. Dually, we consider $\mathcal{U}^{\prime}\left(\mathfrak{s o}_{3,1}\right)$ and $\mathcal{U}^{\prime}$ (poinc) (see Examples 3.13 and 4.4).

We are now ready to construct superextensions. We start by considering the 4-dimensional comodule of $\operatorname{Spin}^{\prime}(3,1)$ with basis $\left\{\theta_{+}, \theta_{-}, \overline{\theta_{+}}, \overline{\theta_{-}}\right\}$and 
left coaction in the obvious way. It gives rise to a braided commutative comodule Hopf algebra $\Theta_{4}$ as in Proposition 6.15. Dually we can consider a 4-dimensional module of $\mathcal{U}^{\prime}\left(\mathfrak{s o}_{3,1}\right)$ with basis $\left\{Q_{+}, Q_{-}, \overline{Q_{+}}, \overline{Q_{-}}\right\}$and action given as in Example 6.19. We can view it as the envelope $\mathcal{U}\left(\omega_{4}\right)$ of the "abelian" super-Lie algebra $\omega_{4}$ with the $Q$ 's forming its basis (i.e. $Q_{i} Q_{j}=$ $\left.-Q_{j} Q_{i}\right)$. Both give immediately rise to semidirect superextensions $\Theta_{4} \rtimes$ $\operatorname{Spin}^{\prime}(3,1)$ and $\mathcal{U}\left(\omega_{4}\right) \rtimes \mathcal{U}^{\prime}\left(\mathfrak{s o}_{3,1}\right)$. Furthermore, by the induced coaction of Poinc' respectively the induced action of $\mathcal{U}^{\prime}(\mathfrak{p o i n c})$ we obtain semidirect superextensions $\Theta_{4} \rtimes$ Poinc $^{\prime}$ and $\mathcal{U}\left(\omega_{4}\right) \rtimes \mathcal{U}^{\prime}(\mathfrak{p o i n c})$.

However, the usual Poincaré super-Lie algebra and supergroup are obtained as follows.

Example 6.30. Consider the graded commutative Spin' $(3,1)$-comodule Hopf algebra SMink built on the tensor product Mink $\otimes \Theta_{4}$ and defined as follows. It has the tensor product algebra structure and the coalgebra structure as for $\Theta_{4}$ and

$$
\Delta x^{\mu}=x^{\mu} \otimes 1+1 \otimes x^{\mu}+\sum_{i, j} \overline{\theta_{i}} \sigma_{i j}^{\mu} \otimes \theta_{j}+\sum_{i, j} \theta_{i} \overline{\sigma_{i j}^{\mu}} \otimes \overline{\theta_{j}} .
$$

on $x^{\mu}$. The antipode is given by $\mathrm{S} \theta_{i}=-\theta_{i}$ and $\mathrm{S} x^{\mu}=-x^{\mu}$.

SMink is precisely (the algebra of functions on) the super-translation group. The super-Poincaré group is now obtained analogous to the ordinary one, namely as the bosonization SPoinc ${ }^{\prime}=\operatorname{SMink} \rtimes \operatorname{Spin}^{\prime}(3,1)$. Explicitly, it is generated as an algebra by $\left\{t_{i j}, \overline{t_{i j}}, x^{\mu}, \theta_{ \pm}, \overline{\theta_{ \pm}}\right\}$. It has the relations $\theta_{i} \theta_{j}=-\theta_{j} \theta_{i}$ and $\theta_{i} t_{j k}=-t_{j k} \theta_{i}$ and all other relations commutative. It has the matrix coalgebra structure on $T$ while for $x^{\mu}$ and $\theta_{i}$ it is given by

$$
\begin{gathered}
\Delta \theta_{i}=\theta_{i} \otimes 1+\sum_{j} t_{i j} \otimes \theta_{j}, \\
\Delta x^{\mu}=x^{\mu} \otimes 1+\sum_{\nu} \Lambda^{\mu \nu} \otimes x^{\nu}+\sum_{i, j, k} \overline{\theta_{i}} \sigma_{i j}^{\mu} t_{j k} \otimes \theta_{k}+\sum_{i, j, k} \theta_{i} \overline{\sigma_{i j}^{\mu}} \overline{t_{j k}} \otimes \overline{\theta_{k}} .
\end{gathered}
$$

Note that SMink is at the same time a quantum homogeneous space (see Remark 6.4) and carries a left coaction of SPoinc' as an algebra by construction. It is thus nothing but super-Minkowski space and gives rise to a quantum principal bundle (SPoinc', SMink, Spin' $(3,1)$ ) which we can view as its spin-bundle (see the remarks in Examples 6.21 and 6.29).

In fact, SMink can be viewed as a braided cocycle extension

$$
\Theta_{4} \hookrightarrow \text { SMink } \rightarrow \text { Mink }
$$

(in the category of $\operatorname{Spin}^{\prime}(3,1)$-comodules) with the injection and surjection defined in the obvious ways. The cocycle Mink $\rightarrow \Theta_{4} \otimes \Theta_{4}$ which determines the extension is given by

$$
x^{\mu} \mapsto \sum_{i, j} \overline{\theta_{i}} \sigma_{i j}^{\mu} \otimes \theta_{j}+\sum_{i, j} \theta_{i} \overline{\sigma_{i j}^{\mu}} \otimes \overline{\theta_{j}} .
$$


For details of the relevant Hopf algebra extension theory we refer the reader to [5]. (The generalization to the $Z_{2}$-grading is straightforward in the present case.)

We can equally utilize the enveloping algebra picture. The sequence (19) becomes after dualization and restriction to the (super) Lie algebras

$$
\mathfrak{t r}_{4} \hookrightarrow \mathfrak{s t r}_{4} \rightarrow \omega_{4} .
$$

Now $\mathfrak{s t r}_{4}$ is a central (graded) extension of $\omega_{4}$ by $\mathfrak{t r}_{4}$. Here, the action of $\mathcal{U}^{\prime}\left(\mathfrak{s o}_{3,1}\right)$ on $\omega_{4}$ is given as in Example 6.19, with the same action for the barred generators and the action between barred and un-barred generators zero. The extension is determined by the graded cocycle $\omega_{4} \otimes \omega_{4} \rightarrow \mathfrak{t r}_{4}$ given by

$$
\overline{Q_{i}} \otimes Q_{j} \mapsto 2 \sum_{\mu} \sigma_{i j}^{\mu} P^{\mu}
$$

This is the familiar way to look at the super-translation Lie algebra $\mathfrak{s t r}_{4}$, see e.g. [21]. The enveloping Hopf algebra version of this is precisely the dual of (20) and lives in the category of $\mathcal{U}^{\prime}\left(\mathfrak{s o}_{3,1}\right)$-modules.

Example 6.31. $\mathfrak{s t r}_{4}$ is the $\mathcal{U}^{\prime}\left(\mathfrak{s o}_{3,1}\right)$-module super-Lie algebra built on the space $\omega_{4} \oplus \mathfrak{t r}_{4}$ with bracket

$$
\left\{\overline{Q_{i}}, Q_{j}\right\}=2 \sum_{\mu} \sigma_{i j}^{\mu} P^{\mu}
$$

and all brackets involving $P^{\mu}$ vanishing. The ordinary super-Poincaré Lie algebra is now the semidirect product $\mathfrak{s t r}_{4} \rtimes \mathfrak{s o}_{3,1}$. However, the proper quantum group (determining the physical symmetries) is the "enveloping" Hopf algebra $\mathcal{U}^{\prime}(\mathfrak{s p o i n c}):=\mathcal{U}\left(\mathfrak{s t r}_{4}\right) \rtimes \mathcal{U}^{\prime}\left(\mathfrak{s o}_{3,1}\right)$. Note that it differs from $\mathcal{U}\left(\mathfrak{s t r}_{4} \rtimes \mathfrak{s o}_{3,1}\right)$ which can be constructed as a graded enveloping Hopf algebra in that it takes into account the spin-statistics relation as explained above. The cross-relations between $\{E, F, H\}$ and $\left\{Q_{ \pm}\right\}$are as in Example 6.19 and extended to the barred generators in the obvious way. The coproducts for all generators are primitive, except for $\Delta Q_{i}=Q_{i} \otimes 1+\xi \otimes Q_{i}$.

Due to Lemma 3.11 SPoinc' and $\mathcal{U}^{\prime}(\mathfrak{s p o i n c})$ are dual (co)triangular Hopf algebras. This induces a natural quantum tangent space which can be identified with spoinc (i.e., it is the smallest quantum tangent space that contains poinc). The derivatives are the ones of Example 6.11 supplemented by

$$
\begin{gathered}
\partial_{H}\left(\theta_{ \pm}\right)= \pm \theta_{ \pm}, \quad \partial_{\bar{H}}\left(\overline{\theta_{ \pm}}\right)= \pm \overline{\theta_{ \pm}}, \quad \partial_{E}\left(\theta_{+}\right)=\theta_{-}, \quad \partial_{\bar{E}}\left(\overline{\theta_{+}}\right)=\overline{\theta_{-}} \\
\partial_{F}\left(\theta_{-}\right)=\theta_{+}, \quad \partial_{\bar{F}}\left(\overline{\theta_{-}}\right)=\overline{\theta_{+}}, \quad \partial_{Q_{i}}\left(\theta_{j}\right)=\delta_{i j}, \quad \partial_{\overline{Q_{i}}}\left(\overline{\theta_{j}}\right)=\delta_{i j} \\
\partial_{Q_{i}}\left(x^{\mu}\right)=\sum_{j} \overline{\sigma_{i j}^{\mu}} \overline{\theta_{j}}, \quad \partial_{\overline{Q_{i}}}\left(x^{\mu}\right)=\sum_{j} \sigma_{i j}^{\mu} \theta_{j} .
\end{gathered}
$$

All other derivatives of generators vanish. 


\section{Conclusions and Outlook}

We have exhibited here a categorial point of view on quantum field theory yielding a generalized notion of symmetry based on quantum groups and braided categories. This is motivated by the observation that rather than symmetry groups themselves, only their representation categories are operationally relevant in quantum field theory. The resulting framework unifies the concepts of conventional symmetry and exchange statistics (as was already noticed in [6]). We have shown how (super)group symmetry, Bose-Fermi statistics and the spin-statistics relation are interconnected in a three-layer structure that recovers the generalized quantum group symmetry of quantum field theory.

Rephrasing the old question of non-trivially extending space-time symmetries in the new framework naturally leads to supersymmetry (assuming Bose-Fermi statistics). Furthermore, we were able to show that (in this framework) supersymmetry is indeed the most general way of unifying external and internal symmetries. Even if we drop the non-triviality condition only group symmetries and supersymmetries are allowed. This appears to be a no-go theorem for "hidden" (non-triangular) quantum group symmetries in physically interesting theories such as the standard model.

However, this has to be interpreted with care. A crucial ingredient in our formulation is the condition that the quantum group which extends the given space-time-statistics quantum group does not modify the statistics. (That is, the cotriangular structure is preserved by the extension). We see this as a natural constituent of the extension problem (and it is implicit in its conventional formulation). For example, it would be conceivable that there exist multiplets of states with braid statistics of which so far only (bosonic or fermionic) singlets have been observed. But as this goes in a sense beyond ordinary quantum field theory it also goes beyond our formulation of the extension problem. Furthermore, the braiding is defined for all objects in the relevant category while not all of them can be necessarily interpreted as being subject to some exchange statistics in the conventional sense. It is thus conceivable that the braiding can have a broader meaning in general and just reduce on the relevant objects to the conventional statistics. This would also leave open the possibility for non-triangular quantum group symmetries.

We have also seen that for theories with non-symmetric braid statistics (e.g. anyons in two spatial dimensions), the separation between the conventional notions of symmetry and statistics can no longer be retained (see the end of Section 2.1 and the beginning of Section 4.3). Only the generalized notion of quantum group symmetry remains applicable. It is thus no surprise that (non-triangular) quantum groups are indeed employed in the construction of fractional supersymmetry (which implies non-symmetric braid statistics) [11, 12.

Finally, we mention that there is a generalization of quantum field theory 
22] to precisely the categorial framework (braided categories) we outline in Section 2.1. This naturally takes in quantum group symmetries and for the proper Poincaré quantum group reconstructed in Section 4.1 yields automatically the correct differences for path integrals and Feynman rules between bosons and fermions [6]. In this context the present paper clarifies how supersymmetric theories would have to be constructed in this framework (namely through their proper quantum group versions considered here). Furthermore, even $q$-deformations of supersymmetries (of which some examples have been considered in the literature, see e.g. [8]) can thus be employed. As $q$-deformation has been proven to be a potential regulator of quantum field theory 22] this yields the prospect of a (notoriously difficult) covariant regularization of supersymmetric theories.

\section{Acknowledgements}

I would like to thank H. Pfeiffer, T. Schücker and F. Girelli for their careful reading and many helpful comments on the manuscript. This work was supported through a NATO postdoctoral fellowship.

\section{References}

[1] S. Coleman and J. Mandula, All Possible Symmetries of the S Matrix, Phys. Rev. 159 (1967), 1251-1255.

[2] Yu. A. Golfand and E. P. Likhtman, Extension of the algebra of Poincaré group generators and violation of $P$ invariance, JETP Lett. 13 (1971), 323-326.

[3] J. Wess and B. Zumino, Supergauge transformations in four dimensions, Nucl. Phys. B 70 (1974), 39-50.

[4] R. Haag, T. Łopuszański, and M. Sohnius, All Possible Generators of Supersymmetries of the S-Matrix, Nucl. Phys. B 88 (1975), 257-274.

[5] S. Majid, Foundations of Quantum Group Theory, Cambridge University Press, Cambridge, 1995.

[6] R. Oeckl, The quantum geometry of spin and statistics, J. Geom. Phys. 39 (2001), 233-252.

[7] J. F. Cornwell, Group theory in physics, vol. 1 \& 2, Techniques of Physics, no. 7, Academic Press, London, 1984.

[8] Yu. I. Manin, Multiparametric Quantum Deformation of the General Linear Supergroup, Commun. Math. Phys. 123 (1989), 163-175. 
[9] S. Majid, Cross Products by Braided Groups and Bosonization, J. Algebra 163 (1994), 165-190.

[10] S. Majid, Braided matrix structure of the Sklyanin algebra and of the quantum Lorentz group, Commun. Math. Phys. 156 (1993), 607-638.

[11] R. S. Dunne, A. J. Macfarlane, J. A. de Azcárraga, and J. C. Pérez Bueno, Geometrical foundations of fractional supersymmetry, Int. J. Mod. Phys. A 12 (1997), 3275-3305.

[12] R. S. Dunne, A braided interpretation of fractional supersymmetry in higher dimensions, J. Math. Phys. 40 (1999), 1180-1196.

[13] A. Klimyk and K. Schmüdgen, Quantum Groups and Their Representations, Springer Verlag, Berlin, 1997.

[14] T. Brzeziński and S. Majid, Quantum Group Gauge Theory on Quantum Spaces, Commun. Math. Phys. 157 (1993), 591-638.

[15] S. Kobayashi and K. Nomizu, Foundations of Differential Geometry, vol. 1, Interscience Publishers, New York, 1963.

[16] S. L. Woronowicz, Differential Calculus on Compact Matrix Pseudogroups (Quantum Groups), Commun. Math. Phys. 122 (1989), 125170 .

[17] S. Majid, Classification of Bicovariant Differential Calculi, J. Geom. Phys. 25 (1998), 119-140.

[18] B. DeWitt, Supermanifolds, Cambridge University Press, Cambridge, 1984.

[19] B. Kostant, Graded manifolds, graded Lie theory, and prequantization, Differential geometrical methods in mathematical physics (Bonn, 1975), Lecture Notes in Math., no. 570, Springer, Berlin, 1977, pp. 177-306.

[20] G. Landi and G. Marmo, Extensions of Lie superalgebras and supersymmetric abelian gauge fields, Phys. Lett. B 193 (1987), 61-66.

[21] J. A. de Azcárraga and J. M. Izquierdo, Lie groups, Lie algebras, cohomology and some applications in physics, Cambridge University Press, Cambridge, 1995.

[22] R. Oeckl, Braided Quantum Field Theory, Commun. Math. Phys. 217 (2001), 451-473. 